\title{
Reconstruction of the lipid metabolism for the microalga Monoraphidium neglectum from its genome sequence reveals characteristics suitable for biofuel production
}

Christian Bogen, Arwa Al-Dilaimi, Andreas Albersmeier, Julian Wichmann, Michael Grundmann, Oliver Rupp, Kyle J Lauersen, Olga Blifernez-Klassen, Jörn Kalinowski, Alexander Goesmann, Jan H Mussgnug and Olaf Kruse*

\begin{abstract}
Background: Microalgae are gaining importance as sustainable production hosts in the fields of biotechnology and bioenergy. A robust biomass accumulating strain of the genus Monoraphidium (SAG 48.87) was investigated in this work as a potential feedstock for biofuel production. The genome was sequenced, annotated, and key enzymes for triacylglycerol formation were elucidated.

Results: Monoraphidium neglectum was identified as an oleaginous species with favourable growth characteristics as well as a high potential for crude oil production, based on neutral lipid contents of approximately 21\% (dry weight) under nitrogen starvation, composed of predominantly C18:1 and C16:0 fatty acids. Further characterization revealed growth in a relatively wide $\mathrm{pH}$ range and salt concentrations of up to $1.0 \% \mathrm{NaCl}$, in which the cells exhibited larger structures. This first full genome sequencing of a member of the Selenastraceae revealed a diploid, approximately 68 Mbp genome with a G + C content of 64.7\%. The circular chloroplast genome was assembled to a 135,362 bp single contig, containing 67 protein-coding genes. The assembly of the mitochondrial genome resulted in two contigs with an approximate total size of $94 \mathrm{~kb}$, the largest known mitochondrial genome within algae. 16,761 protein-coding genes were assigned to the nuclear genome. Comparison of gene sets with respect to functional categories revealed a higher gene number assigned to the category "carbohydrate metabolic process" and in "fatty acid biosynthetic process" in M. neglectum when compared to Chlamydomonas reinhardtii and Nannochloropsis gaditana, indicating a higher metabolic diversity for applications in carbohydrate conversions of biotechnological relevance.

Conclusions: The genome of M. neglectum, as well as the metabolic reconstruction of crucial lipid pathways, provides new insights into the diversity of the lipid metabolism in microalgae. The results of this work provide a platform to encourage the development of this strain for biotechnological applications and production concepts.
\end{abstract}

Keywords: M. neglectum genome, Biofuels, Lipid metabolism, Neutral lipid accumulation

\section{Background}

Phototrophic microalgae are increasingly investigated for their use in biotechnological applications as these unicellular organisms offer the opportunity of introducing sustainable production pathways by converting sunlight energy and $\mathrm{CO}_{2}$ into valuable products [1-5]. In order to establish highly efficient strains, however, the systematic

\footnotetext{
* Correspondence: Olaf.kruse@uni-bielefeld.de

Department of Biology/Center for Biotechnology, Bielefeld University, Universitätsstrasse 27, Bielefeld 33615, Germany
}

genome analysis and the development of molecular tools for genetic engineering approaches are necessary. Consequently the genomes of a number of strains of interest have recently been sequenced and essential genetic tools have been successfully introduced [6-8].

A particular focus has been placed on species with high oil content. Many microalgae are reported to produce considerable amounts of oils [2,3], where the compound of interest for biofuels is mainly triacylglycerol (TAG) [2]. TAG consists of three fatty acids esterified to
C Biomed Central 
a glycerol backbone [3,5]. The accumulation of TAG in microalgae usually occurs under environmental stress conditions such as high-light or nitrogen starvation [2,9] but, however, can be also increased artificially, for example, by inhibition of starch synthesis [10]. Though the lipid metabolism in algae and plants is considered to be comparatively similar, a number of differences can be found. For example, in contrast to unicellular microalgae, TAG synthesis in plants predominantly takes place in specialized tissues or organs [2]. Furthermore, current results indicate not only the presence of clear differences between plants and microalgae, but also between different microalgal species [11]. Microalgae exhibit high levels of diversity between species due to their different evolutionary history, thus it is expected that the lipid metabolism amongst the various strains also exhibits distinct differences. This is exemplified by the comparison of the green algal model organism Chlamydomonas reinhardtii with Nannochloropsis [11]. Variable lipid metabolism within the microalgae is also suggested by the high diversity of lipids of different classes and unusual fatty acids found in individual algae strains, even among the same division [12,13].

Based on this knowledge, numerous systematic screens aiming to identify microalgal species with high lipid content have been performed during recent years [14-19]. With the identification of strains of interest by these approaches, the necessity for systematic analyses of genomes by next-generation sequencing, annotation and reconstruction of lipid metabolic pathways becomes evident. These strategies allow deeper insights into the lipid metabolism and evolutionary strategies of these photosynthetic microorganisms.

The genome of the green algal model organism C. reinhardtii has been sequenced [8], as well as the genome of the TAG-accumulating Eustigmatophytes Nannochloropsis gaditana [20,21], Nannochloropsis oceanica [6], and several other microalgae [22].

In our laboratory, we recently succeeded in identifying a strain of the genus Monoraphidium with high fatty acid abundances combined with robust biomass accumulation through a screening strategy which combined up-scaling tests, determination of total lipids, and the evaluation of fatty acid abundances [19]. The identification of Monoraphidium contortum from this screening is in coincidence with rising interest in this genus over recent years [23,24]. Strains of the genus Monoraphidium belong to the family of the Selenastraceae within the class Chlorophyceae. So far, little information is available on genomes of members of the family Selenastraceae and related species such as Scenedesmus obliquus. In the light of the fact that the biodiversity of microalgae is tremendous, much of the potential for strain identification and characterisation to contribute to liquid biofuel purposes remains to be explored [2].
Based on 18S rRNA analysis, it was shown that species of this genus tightly cluster with species of Ankistrodesmus, Kirchneriella, and other genera, thus not forming a monophyletic group [25]. When $M$. contortum, isolated from a natural habitat in Thailand, was grown in different autotrophic media, it showed comparatively high lipid productivities among the strains under the investigated conditions [23].

Monoraphidium neglectum was also identified as a robust strain belonging to the family Selenastraceae, by our previous screening efforts [19]. M. neglectum was shown to cluster differently to $M$. contortum [24], on the same branch as Podohedriella falcata [25], also known as Ankistrodesmus falcatus. Another strain of this species was reported as an oleaginous organism before [26], highlighting the need for more detailed comparisons and investigations among the members of the Selenastraceae by metabolomic studies and whole genome sequencing. When directly compared to $M$. contortum, $M$. neglectum showed some phenotypical characteristics such as higher robustness in up-scale cultivations, making this strain even more interesting for biotechnological applications.

In this work, we intensively characterised the oleaginous phenotype of Monoraphidium neglectum and established and annotated a draft genome of this organism as a precondition for metabolic network reconstruction. Investigation of the reconstructed metabolic pathways with respect to key enzymes for triacylglycerol formation was carried out, setting the basis for further investigations and offering the possibility to develop strategies for genetic improvements. In addition, $M$. neglectum was further biochemically characterised as a robust production strain suitable for biotechnological approaches.

\section{Results}

The species $M$. neglectum was identified in a previous screen for oleaginous microalgal strains that exhibited robust growth characteristics at varying cultivation conditions [19]. To evaluate the biotechnological potential of this strain, a detailed investigation was performed in this work. This investigation included detailed physiological analysis, evaluating lipid yields and robustness. Genome sequencing and annotation were performed and combined with the reconstruction of phylogeny as well as an analysis of lipid metabolism pathways, features crucial for understanding and establishing M. neglectum as lipid-producing feedstock.

\section{M. neglectum exhibits a rapid photoautotrophic growth phenotype and accumulates neutral lipids to a high extent under nitrogen starvation}

The combination of efficient phototrophic biomass accumulation and high neutral lipid content is considered as 
one of the most essential traits of an algal strain used for liquid biofuel production [17]. Besides nutrient starvation, light stress is an important trigger in the generation of triacylglycerols [2]. Therefore, both factors were investigated in a combined approach. As in previous work, the model alga C. reinhardtii was used as a control. Cells of both species were inoculated to the same biomass density and cultivated phototrophically for three days to obtain comparable starter cultures (Figure 1, precultures). After three days, cells were harvested and used for comparative analyses. To assess lipid accumulation induced by nutrient starvation, cells were resuspended in nitrogen replete medium or in medium deficient in nitrogen (Figure $1,+\mathrm{N}$ or $-\mathrm{N}$, respectively). To assess additional effects related to light availability, two cell densities were chosen for the $-\mathrm{N}$ samples and designated "low density" or "high density", respectively.

For $M$. neglectum, low density cultures corresponded to $0.22 \mathrm{~g} \mathrm{l}^{-1}\left(2 \times 10^{6} \pm 5 \times 10^{5}\right.$ cells ml $\left.{ }^{-1}\right)$ and high density cultures corresponded to $0.78 \mathrm{~g} \mathrm{l}^{-1}\left(6 \times 10^{6} \pm 2 \times 10^{6}\right.$ cells $\mathrm{ml}^{-1}$ ), For the reference strain Chlamydomonas reinhardtii, similar cell densities were chosen. Biomass accumulation was subsequently monitored and lipid contents as well as fatty acid compositions were determined after five days of cultivation (Figure 1).

The neutral lipid content (per dry weight) increased from initial $1.4 \pm 0.6 \%(+\mathrm{N}$, preculture) to up to $20.9 \pm$ $4.0 \%$ upon nitrogen starvation in low-density cultures in M. neglectum (Figure 1B).
The overall neutral lipid productivity was determined during the two-step cultivation process for the high density cultures. In this condition, $M$. neglectum reached maximal productivity rates of $52 \pm 6 \mathrm{mg}$ neutral lipids $\mathrm{l}^{-1}$ day $^{-1}$, which was four times higher compared to C. reinhardtii $\left(13 \pm 4 \mathrm{mg}\right.$ neutral lipids $\mathrm{l}^{-1}$ day $^{-1}$, see Additional file 1: Figure S1), a finding that could, in particular, be attributed to a pronounced increase of biomass in the beginning of the starvation period in the case of M. neglectum. The relative abundance of fatty acids was determined (with an internal C17-FA standard set to a value of 100) to directly compare the individual fatty acid accumulation between $M$. neglectum and $C$. reinhardtii. M. neglectum reached comparatively high overall abundances of fatty acids in the total lipid fraction under nitrogen starvation that were roughly 50\% higher than those detected in C. reinhardtii (Figure 1C, Additional file 2: Figure S2). While the total abundance of fatty acid increased only 1.1-fold in high density and 1.5-fold in low density cultures in $C$. reinhardtii when compared to the nutrient replete control (Figure 1C, Additional file 2: Figure S2), $M$. neglectum showed a 2-fold and 2.5-fold increase of its fatty acid content in high and low density cultures respectively (Figure 1C, Additional file 2: Figure S2). When only the fatty acids of the neutral lipid fraction were considered, this finding became even more evident.

In $M$. neglectum, the fatty acid abundances encountered in the neutral lipid fraction of N-starved cells of

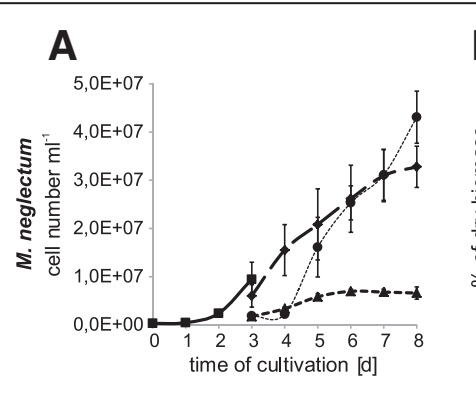

B
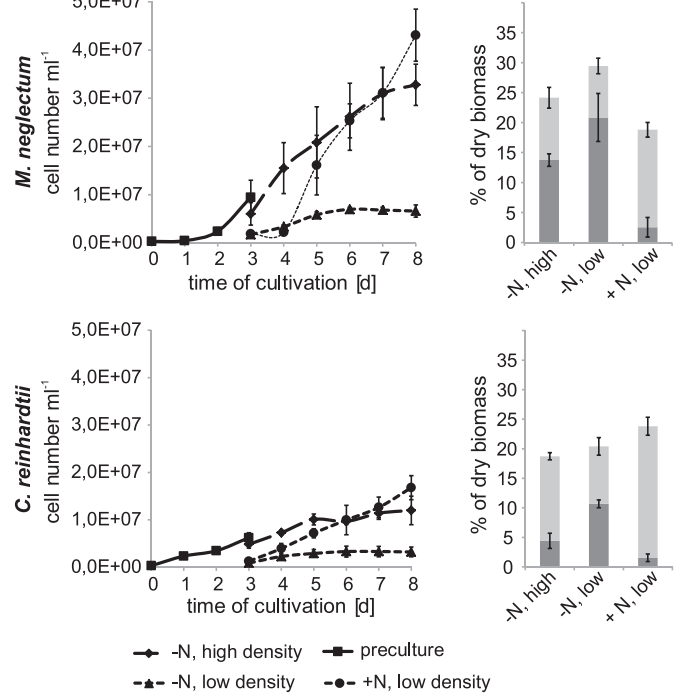

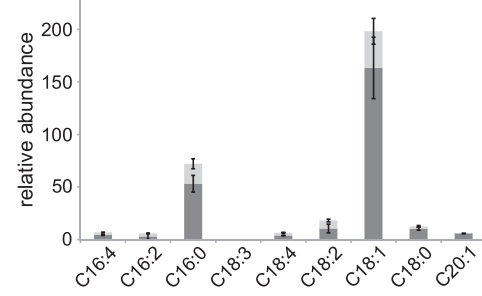

C

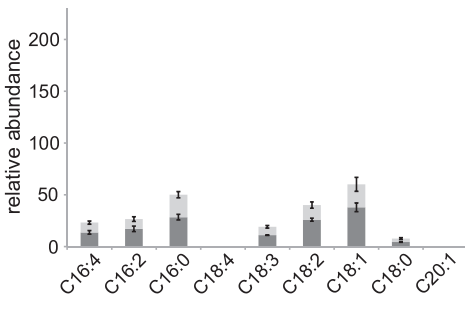

polar lipids $\quad$ neutral lipids

Figure 1 Comparison of growth and lipid accumulation of Monoraphidium neglectum with Chlamydomonas reinhardtii. (A) Growth under nitrogen replete $(+\mathrm{N})$ and nitrogen deficient $(-\mathrm{N})$ conditions at two different culture densities (high and low density) $(n=6)$. (B) Neutral and polar lipid content as determined after modified Folch extraction and subsequent chromatography after five days of cultivation $(n=4)$. (C) Relative abundances of fatty acids (determined by comparison to internal C17-FA standard set to a value of 100) of M. neglectum (top) and C. reinhardtii (bottom) after nitrogen starvation for five days in low culture densities $(-N$, low density), $(n=4)$. Error bars indicate standard deviation. 
low density cultures were about tenfold higher in comparison to those of the non-starved cultures (Figure 1C, Additional file 2: Figure S2). This accumulation pattern is similar to the observation in C. reinhardtii, where fatty acids of the neutral lipid fraction increased more than tenfold (Figure 1C, Additional file 2: Figure S2) in cultures with lower biomass densities when compared to the nutrient replete control. $M$. neglectum, therefore, accumulated neutral lipids and fatty acids to high abundances under nitrogen limitation, a feature which was enhanced with increased light penetration. These cultivation parameters also resulted in a positive effect on the accumulation of fatty acids within the neutral lipid fraction in the investigated strains. Interestingly, both strains exhibited a decrease of fatty acids in the polar lipid fraction when nitrogen starvation at low culture densities was applied (Figure 1C, Additional file 2: Figure S2), an observation also reported for other strains [2].

In $M$. neglectum, the increase of the overall fatty acid content under nitrogen starvation could be mostly attributed to an accumulation of C18:1 and C16:0 which contributed considerably to the neutral lipid fraction (Figure $1 \mathrm{C}$ ). This finding is also reflected by the gravimetrical determinations which show a pronounced increase of the neutral lipid fraction, while the total lipid content increased only moderately (Figure 1B).

In contrast, $C$. reinhardtii demonstrated a more diverse accumulation pattern of fatty acids in the neutral lipid fraction under nitrogen starvation, where not only saturated $\mathrm{C} 16$ and monoenoic $\mathrm{C} 18$ but also C16:4, C18:3 and dienoic $\mathrm{C} 18$ abundances increased (Figure 1C).

It is well established that microalgal biomass and lipid production are strongly dependent on the cultivation conditions [27]. It was, therefore, important to compare the production rates of $M$. neglectum with other known oleaginous species in the chosen setup. Parachlorella kessleri and Scenedesmus obliquus were selected for comparative analyses because they represent prominent microalgal species with superior lipid productivities $[17,28]$. Low density conditions were applied for all strains to achieve maximum lipid accumulation levels (Table 1).
When directly compared, M. neglectum demonstrated the highest overall biomass productivity, exceeding those of all three control strains by $33-68 \%$ under optimal nutrient replete conditions (Table 1).

After nitrogen starvation, $M$. neglectum had a maximal total lipid content of up to $30 \pm 3.4 \%$ of dried biomass, significantly higher than in the control strain C. reinhardtii with only $20 \pm 0.8 \%$ (Table 1 ). The total lipid amount in the oleaginous species $P$. kessleri and S. obliquus was determined to be higher in this setup; however, the neutral lipid content of $M$. neglectum was comparable to the top performer S. obliquus. The results demonstrate that neutral lipid production of $M$. neglectum can be at least equal to $P$. kessleri and S. obliquus. Therefore, $M$. neglectum represents a new oleaginous microalgal species.

\section{M. neglectum shows a robust growth phenotype with a} high salt and $\mathrm{pH}$ tolerance during phototrophic growth The ability to support growth in brackish or marine environments is an important trait for strains considered for large scale outdoor cultivations. Salt tolerance and cellular adaptation reactions were systematically evaluated in terms of growth, lipid content and composition as well as cell morphology. As shown in Figure 2A, salt concentrations of $0.5 \%(\mathrm{w} / \mathrm{v})$ did not affect the biomass accumulation negatively. When salt concentrations were increased to $1 \%$, cells still survived but biomass accumulation was severely reduced.

Neutral lipid contents were found to increase with the induction of salt stress, reaching a maximal content of $8.3 \%$ (dry weight) for the cultures grown at $1 \%$ salt concentration (Figure 2B). Furthermore, a clear effect of the salt concentration on the cell shape and size was found (Figure 2C). While cells exhibited a mace-like shape with an average size of about $10 \mu \mathrm{m}$ at lower salt concentrations, larger round cell clusters of about $25 \mu \mathrm{m}$ were formed and sustained when the salt content of the media increased, potentially mitigating the salt stress by decreasing the cellular surface to volume ratio.

It is well known that the $\mathrm{pH}$ can be affected during prolonged microalgal cell cultivation, for example, by secreted fermentation products. For a production strain,

Table 1 Biomass productivities and lipid content of Monoraphidium neglectum compared to other microalgal species

\begin{tabular}{lcccc}
\hline & $\begin{array}{c}\text { Biomass production } \\
\text { (max) }\left[\mathbf{g}^{-1} \mathbf{~}^{-1} \text { day }\right]\end{array}$ & $\begin{array}{c}\text { Biomass production } \\
\text { (mean) }\left[\mathbf{g ~ l}^{-1} \text { day }^{-1}\right]\end{array}$ & $\begin{array}{c}\text { Lipid contents (non-starved) [\% DW] } \\
\text { Total lipids/Neutral lipids }\end{array}$ & $\begin{array}{c}\text { Lipid content (N-Starvation) [\% DW] } \\
\text { Total lipids/Neutral lipids }\end{array}$ \\
\hline Monoraphidium neglectum & 0.967 & 0.603 & $21 \pm 1.7 / 2.5 \pm 1.6$ & $30 \pm 3.4 / 20.9 \pm 4.0$ \\
Chlamydomonas reinhardtii & 0.575 & 0.363 & $24 \pm 1.4 / 1.5 \pm 0.7$ & $20 \pm 0.8 / 10.7 \pm 0.7$ \\
Parachlorella kessleri & 0.650 & 0.435 & $22.6 / 1.2$ & $33.9 / 18.0$ \\
Scenedesmus obliquus & 0.725 & 0.435 & $17.4 / 1.4$ & $37.9 / 21.5$ \\
\hline
\end{tabular}

Biomass productivities and lipid contents from nitrogen replete and N-starved low density cultures. Standard deviation is shown from 4 replicates for $M$. neglectum and C. reinhardtii, and means of two replicates for P. kessleri and S. obliquus. 
A

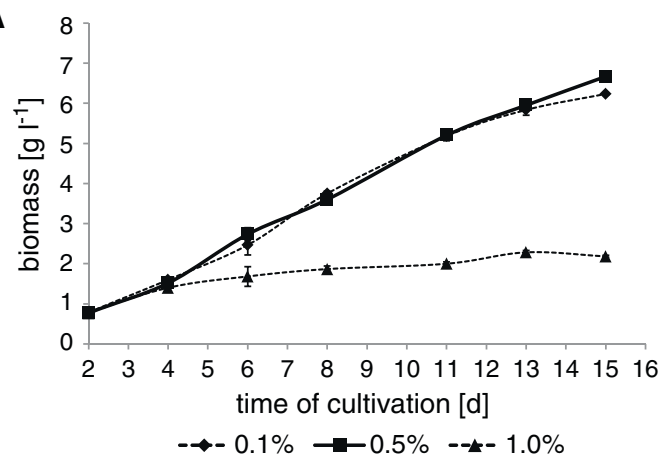

C

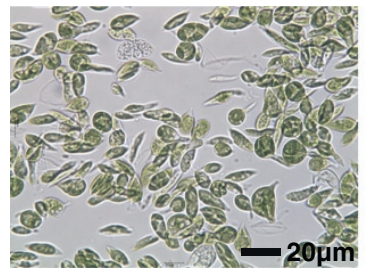

$0.1 \% \mathrm{NaCl} / \mathrm{l}$

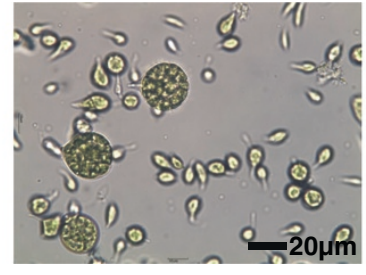

$0.5 \% \mathrm{NaCl} / \mathrm{l}$

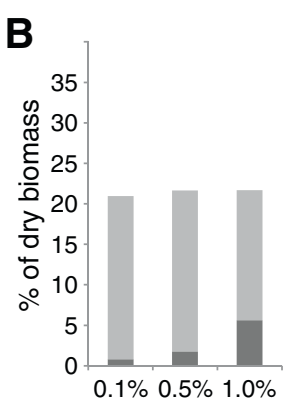

neutral lipids polar lipids

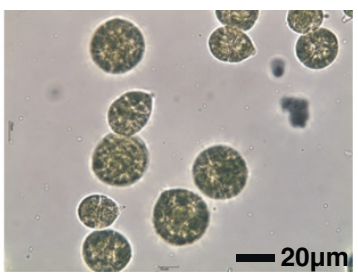

$1.0 \% \mathrm{NaCl} / \mathrm{l}$

Figure 2 Salt tolerance evaluation of $\boldsymbol{M}$. neglectum. Cultivations were performed with $0.1 \%$ (salt concentration of the standard medium), $0.5 \%$ or $1 \%(\mathrm{~W} / \mathrm{V})$ sodium chloride. (A) Biomass accumulation at $350-400 \mu \mathrm{mol}$ photons $\mathrm{m}^{-2} \mathrm{~s}^{-1}$ constant light, error bars indicate standard deviation $(n=3)$. (B) Neutral and polar lipid content in percent of dry biomass of two replicates. (C) Light microscopic images, black bar corresponds to $20 \mu \mathrm{m}$.

tolerance to $\mathrm{pH}$ differences can, therefore, be advantageous. In addition, cultivation at higher $\mathrm{pH}$ increases the efficiency of $\mathrm{CO}_{2}$ fixation and may decrease bacterial contamination under non-sterile cultivation conditions. Plate assays were performed to evaluate the sensitivity of $M$. neglectum to a varying $\mathrm{pH}$ range. $M$. neglectum exhibited tolerance to a wide range of $\mathrm{pH}$ conditions and was able to grow between $\mathrm{pH} 5$ and $\mathrm{pH} 10$ (Figure 3A).

The fact that $M$. neglectum was able to tolerate salt concentrations found in brackish water and was furthermore able to cope with a range of $\mathrm{pH}$ conditions demonstrated its generally robust growth characteristics.

As antibiotics are frequently used in the generation of axenic cultures, as well as selective agents for gene transformation, we investigated whether $M$. neglectum could tolerate antibiotics commonly used for these strategies (Figure 3B). Concentrations of kanamycin, hygromycin $B$, and paromomycin ranging from $10-30 \mu \mathrm{g} \mathrm{ml}^{-1}$ were applied to plate-level cultures under mixotrophic growth. Although all three substances belong to the group of aminoglycoside antibiotics, significant differences regarding cell toxicity were observed. $M$. neglectum demonstrated antibiotic resistance against kanamycin with concentrations up to $30 \mu \mathrm{g} \mathrm{ml}^{-1}$, whereas severe sensitivities to hygromycin B were observed already at $10 \mu \mathrm{g} \mathrm{ml}^{-1}$. The strain also demonstrated resistance to paromomycin. Consequently, powerful antibiotics are available as tools to decontaminate $M$. neglectum cultures (kanamycin, paromomycin) or as selection reagents for future transformation approaches (hygromycin B).

Full genome sequencing and assembly reveals the diploid character of the $M$. neglectum genome

The genome of $M$. neglectum was sequenced by nextgeneration sequencing techniques to elucidate its metabolic pathways and to lay the foundation for a detailed genetic analysis. The genome sequence was obtained using the Illumina $\mathrm{MiSeq}^{\odot}$ technology yielding paired-end reads of $2 \times 250$ bases in length. Over 8 million paired reads were assembled, resulting in about 6,700 scaffolds: thereof 857 were longer than $20 \mathrm{~kb}$ (Table 2).

The investigation of contig-length vs. read-count (Additional file 3: Figure S3) as performed according to [29] revealed a diploid character of the sequenced genome showing homozygous and heterozygous contigs. To identify the contigs and scaffolds constituting the chloroplast and mitochondrion of M. neglectum, a tBLASTx search was carried out using genes of other known chloroplast and mitochondrial genomes. The chloroplast genome was assembled into a single circular contig with a size of 135,362 bp (Figure 4) and a G+C content of $32.38 \%$. The chloroplast genome contains the large single copy (LSC), the small single copy (SSC), as well as inverted repeat (IR) regions (Figure 4). The assembly of the mitochondrial genome led to two contigs separated by gaps (Figure 5). Since the gaps are in-between scaffolded 


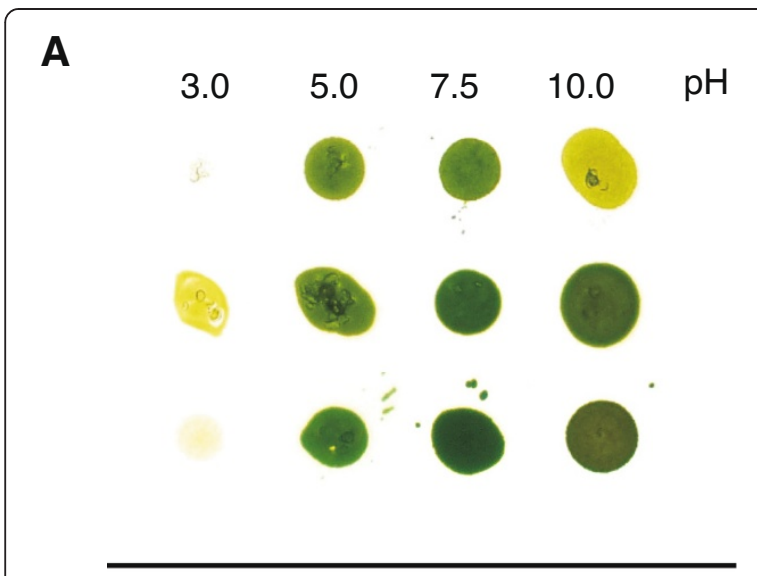

B

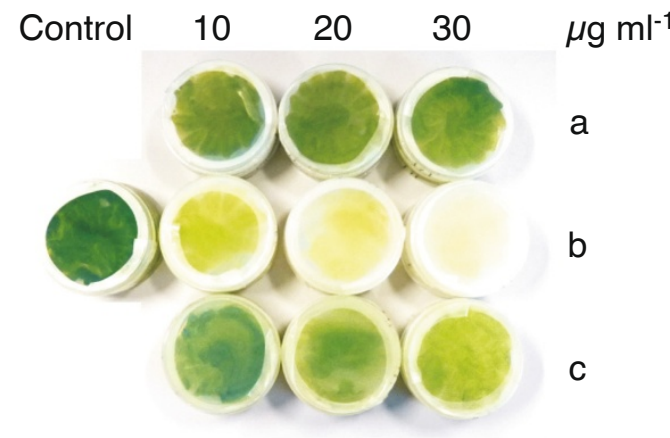

Figure 3 Investigation of growth on M. neglectum at different $\mathrm{pH}$ and with different antibiotics when grown on agar plates under constant illumination. (A) Test of $\mathrm{pH}$ tolerance for $\mathrm{M}$. neglectum. Rows indicate the three biological replicates. (B) Culture sensitivity to antibiotics in ProF media supplemented with $1 \%$ glucose. Three different concentrations of (a) kanamycin, (b) hygromycin and (c) paromomycin, control without antibiotics.

Table 2 Assembly statistics for nuclear draft genome of M. neglectum

\begin{tabular}{ll}
\hline Estimated genome size & $\sim 68 \mathrm{Mb}$ \\
Genomic G + C content & $64.74 \%$ \\
Coverage & 49.30 \\
Aligned reads & $16,194,053$ \\
Number of assembled scaffolds & 6,739 \\
Number of scaffolds $>20$ kb in length & 857 \\
Scaffold N50 & 1,303 \\
Scaffold L50 & 15,659 \\
Number of contigs & 25,211 \\
Number of contigs $>20$ kb in length & 11 \\
Number of contigs $>2$ kb in length & 2103 \\
Scaffolded contig N50 & 2,150 \\
Scaffolded contig L50 & 9,165 \\
\hline
\end{tabular}

contigs, it is certain that the mitochondrial genome is circular. The mitochondrial genome has a size of approximately $94 \mathrm{~kb}$ and a $\mathrm{G}+\mathrm{C}$ content of $45.32 \%$. The remaining scaffolds add to an estimated nuclear genome size of approximately $68 \mathrm{Mb}$ with a $\mathrm{G}+\mathrm{C}$ content of $64.7 \%$ (Table 2).

\section{Genome annotation identifies $M$. neglectum as member of} the Selenastraceae with a large mitochondrial genome Gene prediction was performed with different bioinformatics approaches. Prediction by Augustus [31], trained with the $C$. reinhardtii genome, resulted in 13,930 potential genes and prediction by GeneMark-ES [32] in 20,149. Both predictions were analysed by EVidenceModeler [33], integrating BLASTp protein alignment data with C. reinhardtii. This validation step resulted in a set of 16,845 genes (assuming one transcript per gene) (Table 3).

During validation, $0.2 \%$ of genes predicted by Augustus and $15.4 \%$ of genes predicted by GeneMark were rejected by EVidenceModeler. Visualisation of gene predictions and further manual curation of data were performed using the in-house genome annotation system GenDB [34], modified for eukaryotic genomes (GenDBE).

To investigate the chloroplast-specific features of M. neglectum, the chloroplast genome was compared with those of $C$. reinhardtii and $N$. gaditana (Figure 4, Additional file 4: Figure S4). The chloroplast genome of M. neglectum is similar to $N$. gaditana and C. reinhardtii concerning the gene content. The three chloroplasts have a common set of 77 orthologous genes, including regions encoding ribosomal RNAs and tRNAs (Figure 4, Additional file 4: Figure S4). As in other chloroplast genomes, these genes encode crucial functions of photosynthesis and chloroplast-specific gene expression. Furthermore, all subunits for the synthesis of an active light-independent protochlorophyllide reductase are present.

The unique sets observed for each chloroplast genome include $12 \mathrm{M}$. neglectum-specific genes, $66 \mathrm{~N}$. gaditanaspecific genes, and 18 genes specific to the $C$. reinhardtii chloroplast. Several duplications of genes where found in the $M$. neglectum chloroplast, such as for the B subunit of ATP synthetase, the photosystem $\mathrm{Q}(\mathrm{B})$ protein (psbA), the photosystem I assembly protein (ycf4) and for several tRNAs. In addition, the chloroplast of $M$. neglectum exhibits specific single copy genes, such as the translation initiation factor IF-1 (encoded by infA).

Interestingly, the chloroplast genomes of the three algae have a similar gene composition, but only little synteny could be found.

This finding is supported by the large variance in the chloroplast genome size encountered among sequenced algal genomes [35,36]. In contrast, when compared to the chloroplast genomes of algae, conservation of structure 


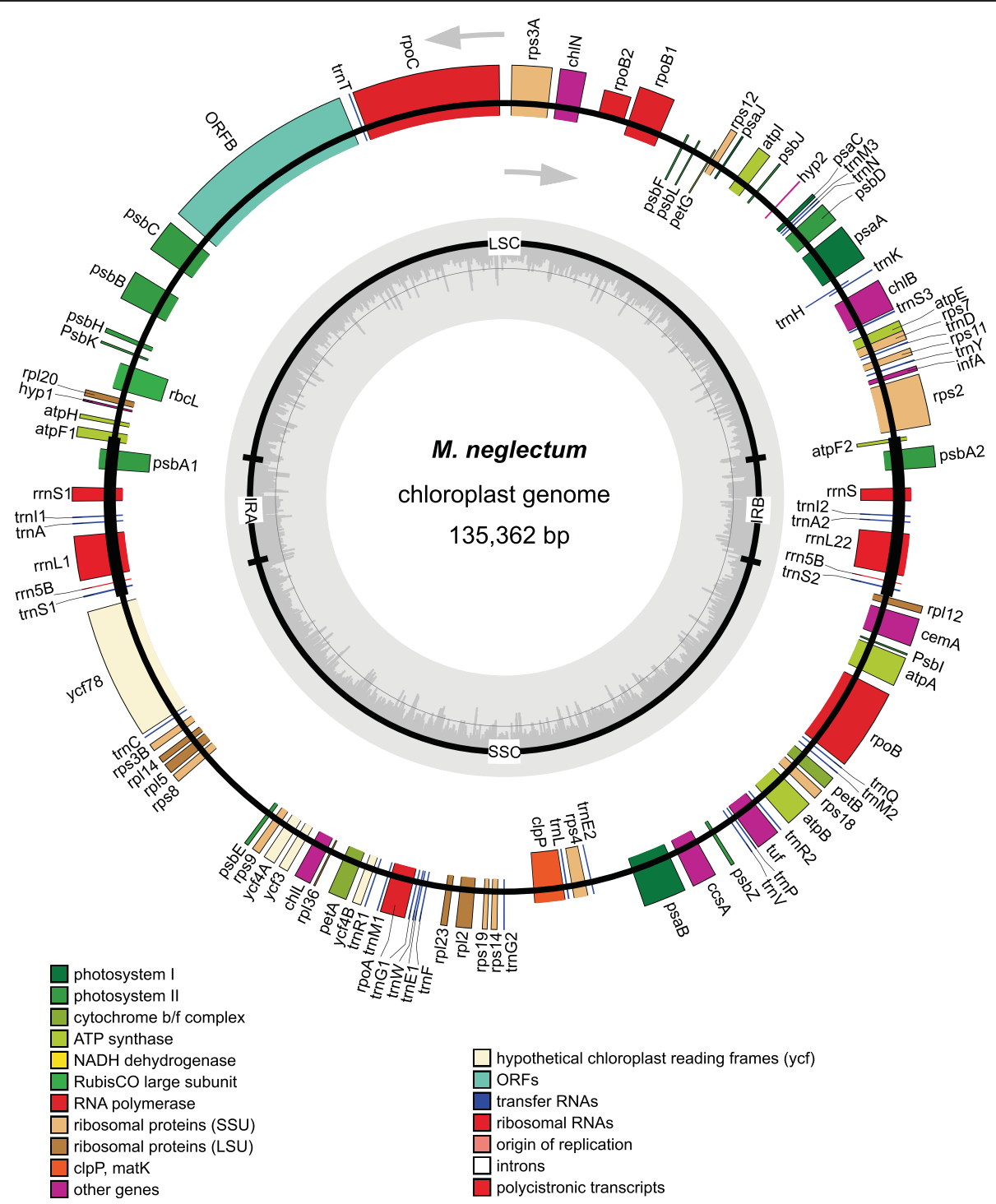

Figure 4 Chloroplast genome of Monoraphidium neglectum. Visualization of the DNA sequence was performed via OrganellarGenomeDRAW (OGDRAW) [30].

and gene content of chloroplasts appears to be greater in plants.

The mitochondrial genome of $M$. neglectum is almost six times as large as the mitochondrial genome of $C$. reinhardtii (16 kb); however, it contains fewer genes and extended, potentially non-coding regions. This observation is in accordance with the understanding that algal mitochondrial genomes generally show a high degree of diversity [37]. In total, 17 genes plus 23 tRNAs were identified in the mitochondrial genome of $M$. neglectum. The basic set of mitochondrial genes is present, including subunits of the NADH dehydrogenase (nad1, nad3, nad4, nad5 and nad6), the cytochrome bc1 complex, cytochrome oxidases (cox2 and cox3) and one subunit of ATP synthase (atp9).
The 23 tRNAs observed represent an almost complete set of tRNA for translation. Only a threonine-tRNA gene is missing in the mitochondrial genome, together with ribosomal RNA genes. It is well known that the expression of mitochondrial genes is mainly dependent on the nucleus-encoded transcriptional and translational machinery [38].

The nuclear genome of $M$. neglectum contains approximately 23 copies of a contig harbouring stable RNA genes including 18S, 28S, and 8S rRNA, as well as 40 tRNAs, and one pseudo-serine tRNA. The 40 tRNAs form a complete set for protein synthesis.

The overall protein coding sequence lengths were compared between the three algal genomes. M. neglectum exhibits a coding sequence length of $3.156 \mathrm{Mb}$, 


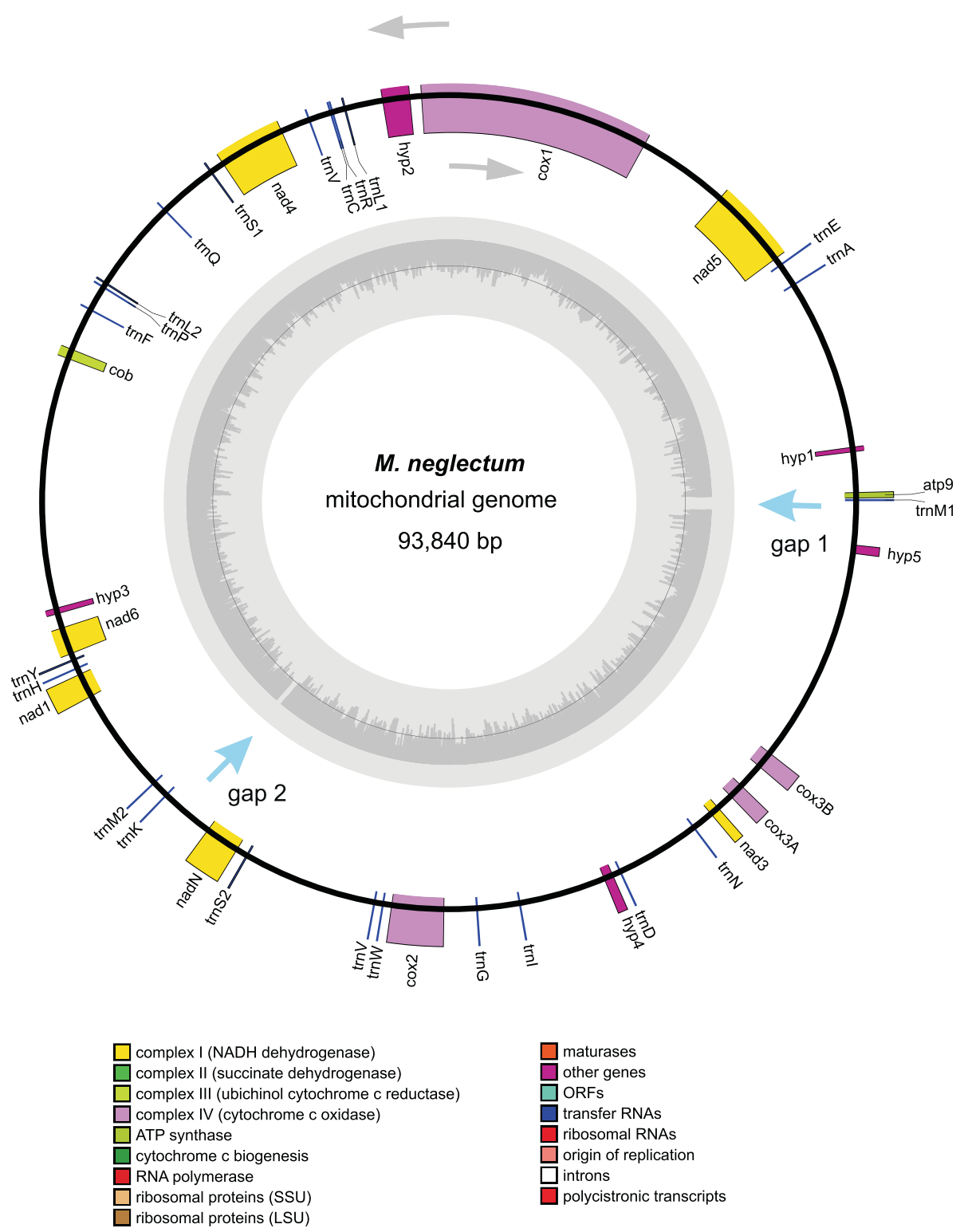

Figure 5 Mitochondrial genome of Monoraphidium neglectum. Visualization of the mitochondrial genome via OrganellarGenomeDRAW (OGDRAW) [30].

approximately $16 \%$ greater than $C$. reinhardtii $(2.7 \mathrm{Mb})$ and even more than $114 \%$ when compared to N. gaditana $(1.473 \mathrm{Mb})$. This number is likely to be reduced by future transcriptional investigations.

To overcome fragmentations, the sequenced Monoraphidium genome was compared with the datasets derived from RNA sequencing approaches of the already well-investigated $C$. reinhardtii and $N$. gaditana. The comparative approach, conducted using EDGAR software [39], taking into account only genes shared between $M$. neglectum and $C$. reinhardtii, yielded 4,249 protein coding regions with an average length of $891 \mathrm{bp}$. In addition, $M$. neglectum and $N$. gaditana share 2,190 protein coding genes with an average length of $748 \mathrm{bp}$. In comparison, 5,945 proteins could be identified as singletons with an average length of only $191 \mathrm{bp}$. This finding indicates that a comparative approach can be used as a powerful tool to reduce substantially potential false positives gene predictions. These assumptions, however, require further confirmation by future RNA sequencing of M. neglectum.

Phylogenetic relationship analyses confirmed that M. neglectum clusters within the family of Selenastraceae (Figure 6), but shows a pronounced difference in its $18 \mathrm{~S}$ rDNA sequence when compared to $M$. braunii [25]. Selenastraceae also cluster closely to the branch of 
Table 3 Gene prediction statistics

\begin{tabular}{|c|c|}
\hline Total number of genes & 16,845 \\
\hline Coding density & $25.16 \%$ \\
\hline Number of nuclear genes & 16,761 \\
\hline Chloroplast genes & 67 \\
\hline Mitochondrial genes & 17 \\
\hline Nuclear rRNA & $\begin{array}{l}\text { ca. } 115 \text { ( } 23 \text { copies of a contig containing } \\
1 \times 8 \mathrm{~S}, 2 \times 28 \mathrm{~S} \text {, and } 2 \times 18 \mathrm{~S})\end{array}$ \\
\hline Chloroplastic rRNA & $6(2 \times 5 S, 2 \times 23 S$, and $2 \times 16 S)$ \\
\hline Mitochondrial rRNA & none \\
\hline Nuclear tRNA & $40+1 \times$ Pseudo Ser-tRNA \\
\hline Chloroplastic tRNA & $29+1 \times$ Pseudo Leu-tRNA \\
\hline Mitochondrial tRNA & $21+1 \times$ Pseudo Met-tRNA \\
\hline $\begin{array}{l}\text { Maximal coding sequence } \\
\text { length }\end{array}$ & $21,519 \mathrm{bp}$ \\
\hline $\begin{array}{l}\text { Minimal coding sequence } \\
\text { length }\end{array}$ & $144 \mathrm{bp}$ \\
\hline $\begin{array}{l}\text { Median coding sequence } \\
\text { length }\end{array}$ & $155 \mathrm{bp}$ \\
\hline Maximal intron length & $7,198 \mathrm{bp}$ \\
\hline Minimal intron length & $23 \mathrm{bp}$ \\
\hline Median intron length & $401 \mathrm{bp}$ \\
\hline Number of single exon genes & 3,030 \\
\hline
\end{tabular}

Scenedesmus obliquus, which was used as an oleaginous control species in this work and had been recommended for liquid biofuel applications before $[17,40]$.

\section{Comparative analyses of the predicted gene functions} revealed higher gene numbers assigned to carbohydrate metabolism and fatty acid biosynthesis

For a deeper investigation of gene functions of $M$. neglectum in comparison to C. reinhardtii and N. gaditana, we calculated the gene ontology terms for the complete gene-sets of the three genomes using InterPro [41].
In general, most functional categories reveal slightly lower numbers of genes in $N$. gaditana, probably reflecting the lower total gene number in this organism (Figure 7). Whereas $C$. reinhardtii shows the highest number of genes in categories such as "protein binding", "DNA binding", and "protein kinase activity", suggesting a larger regulatory repertoire, $M$. neglectum exhibits a higher gene number in the category "catalytic activity". This higher abundance of genes encoding putative metabolic functions is reflected in more detail in the categories "carbohydrate metabolic process" and "fatty acid biosynthetic process", in which, $M$. neglectum again displays the highest gene numbers of the three genomes.

The attribution of predicted genes to gene families here provides a first, general, overview on gene families in $M$. neglectum. This, however, needs to be confirmed by more detailed functional and biochemical investigations. An important point to consider is the elimination of pseudogenes that could be identified by techniques such as transcriptome sequencing.

\section{Detailed genome analysis based on lipid pathway reconstruction provides essential new insights into the lipid metabolism of green microalgae}

Metabolic pathway reconstruction was performed based on the genome data to investigate potential strategies to further improve TAG production. The pathways for fatty acid biosynthesis and the glycerolipid metabolism were reconstructed by comparison of annotated genes with KEGG database assignments. As a result, the key enzymes of the chloroplast fatty acid synthesis pathway have been identified (Figure 8).

These enzymes include the malonyl-CoA:ACP transacylase (MAT, 2.3.1.39), the beta-ketoacyl-acyl-carrierprotein synthase 3 (KAS3, EC number: 2.3.1.180), the beta-ketoacyl-acyl-carrier-protein synthase $1 / 2$ (KAS1/2, EC number: 2.3.1.179), the 3-oxoacyl-ACP reductase (KAR, EC number: 1.1.1.100), the beta-hydroxyacyl-ACP

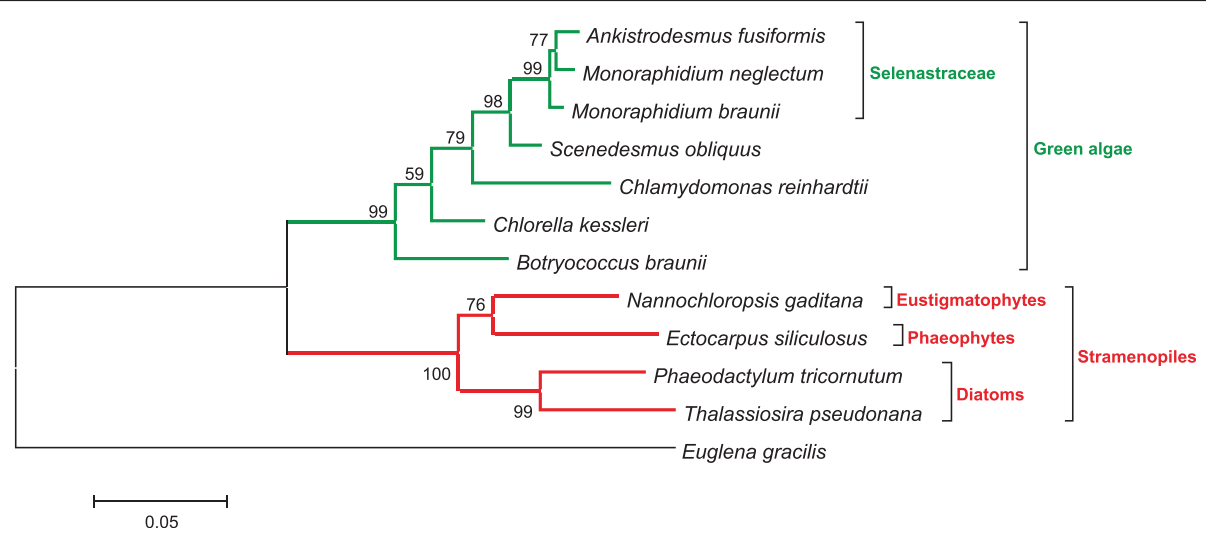

Figure 6 Molecular phylogenetic analysis based on the 18S rDNA sequences using the maximum likelihood method. 


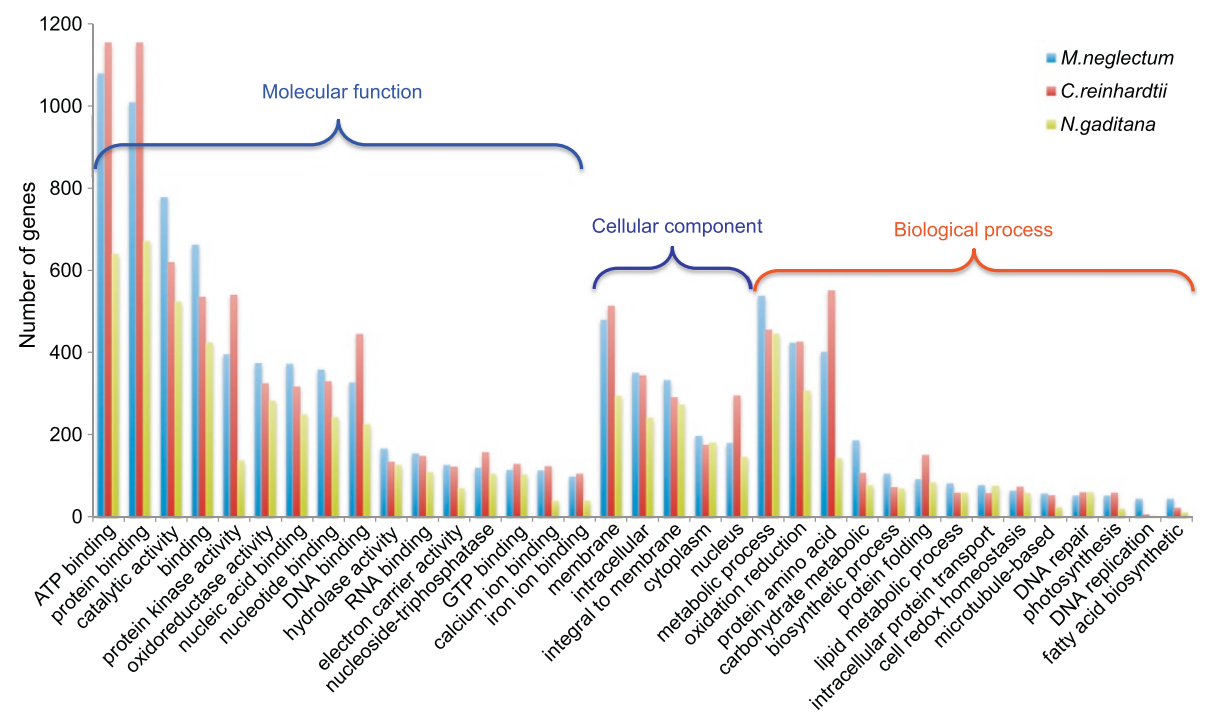

Figure 7 Gene ontology (GO) assignments for $\mathbf{M}$. neglectum, C. reinhardtii, and $\boldsymbol{N}$. gaditana. The 35 most extensive GO-terms of the three GO- super categories "molecular function", "cellular component" and "biological process" are shown. Identification of gene-specific GO-terms was calculated using InterPro [41].

dehydrase (HAD, EC number: 4.2.1.), and an enoyl-ACP reductase (EAR, EC 1.3.1.9).

This set of enzymes is able to catalyse the complete reaction chain from acetyl-CoA via acyl carrier proteins (ACP) to the respective fatty acyl-ACP. Notably, a comparatively high number of homologous genes were identified for acetyl-CoA carboxylase with seven genes (ACC, EC number: 6.4.1.2) and KAR with 11 genes. In addition, homologues have been identified for the fatty acyl-ACP hydrolase (OAH, EC number: 3.1.2.14, PAH, EC number: 3.1.2.22) which cleaves the fatty acid from the acyl carrier protein, releasing the free fatty acid usually as palmitate or stearate, which can then be subjected to several modifications, such as elongation or desaturation. In total, 17 fatty acid desaturase (EC 1.14.19.-) homologues were detected, including five delta-9 desaturase (EC 1.14.19.1) as well as six delta-12 desaturase (EC: 1.14.19.6) homologues.

In addition to the fatty acid metabolism, genes coding for enzymes of the glycerolipid metabolism $[42,43]$ were investigated in more detail. Two genes encoding proteins that show high similarity to acyl-CoA:diacylglycerol acyltransferases type 2 (DGAT2) were identified as well as one encoding a DGAT type 1 homologue. DGAT catalyses the final reaction in TAG generation in the acyl-CoA dependent pathway (Figure 9) and its orthologs have been intensely studied in several phototrophic organisms such as Arabidopsis thaliana, Brassica napus, and C. reinhardtii [44-47]. Other identified genes of this pathway include glycerol kinase (GK, EC 2.7.1.30), glycerol-3-phosphate O-acyltransferase (GPAT, EC 2.3.1.15), 1-acylglycerol-3-phosphate O-acyltransferase (AGPAT, EC number: 2.3.1.51), and phosphatidate phosphatase
(PP, EC number: 3.1.3.4), which are responsible for the formation of lysophosphatidic and phosphatidic acid, as well as diacylglycerol, respectively (Figure 9).

The role of a further acyl-transferase, the phospholipid:diacylglycerol acyltransferase (PDAT), appears highly interesting for TAG metabolism, as reported recently [48]. PDAT mediates an acyl-CoA independent pathway to generate TAG. It was shown that PDAT of $C$. reinhardtii is able to utilise multiple substrates and performs various enzymatic reactions, ranging from TAG synthesis via transacylation of DAG with phospholipid or galactolipid acyl groups to transacylation of two DAG [48].

PDAT was shown not only to be able to hydrolyse phospholipids, galactolipids and cholesteryl esters but also TAG, therefore, attributing an important function in membrane turnover as well as TAG synthesis and degradation to this enzyme. When the genome of $M$. neglectum was analysed for PDAT homologues, two candidates were found, with one (Mneg_03131.t1) showing a conserved phosphatidylcholine-sterol O-acyltransferase domain (Figure 9), representing interesting potential targets for future biotechnological engineering approaches.

Since $M$. neglectum exhibits a strong response to nitrogen depletion, the regulation of genes involved in TAG generation as well as in the breakdown of polar lipids is of special interest. A nitrogen response regulator (NRR) has been identified in the genome of C. reinhardtii and was shown to be involved in neutral lipid accumulation under nitrogen depletion, as well as in the regulation of DGAT type 1 and type 2 expression [45]. A potential homologue of this regulator was found in the genome of M. neglectum (Mneg_07163.t1) which also exhibits a 


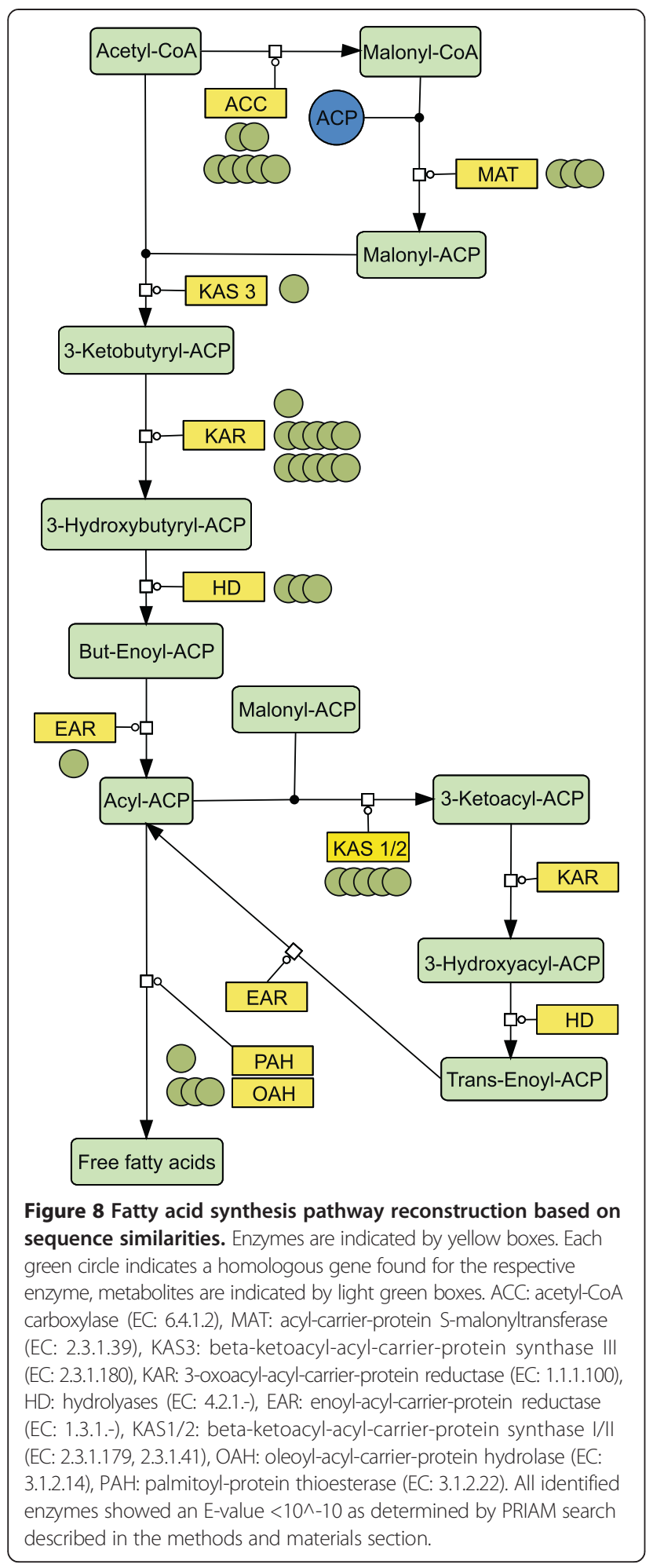

putative SQUAMOSA promoter-binding-like domain, and could, therefore, play a role in the nitrogen stress response of $M$. neglectum.

The successful reconstruction of the metabolic pathways of fatty acid and glycerolipid synthesis clearly

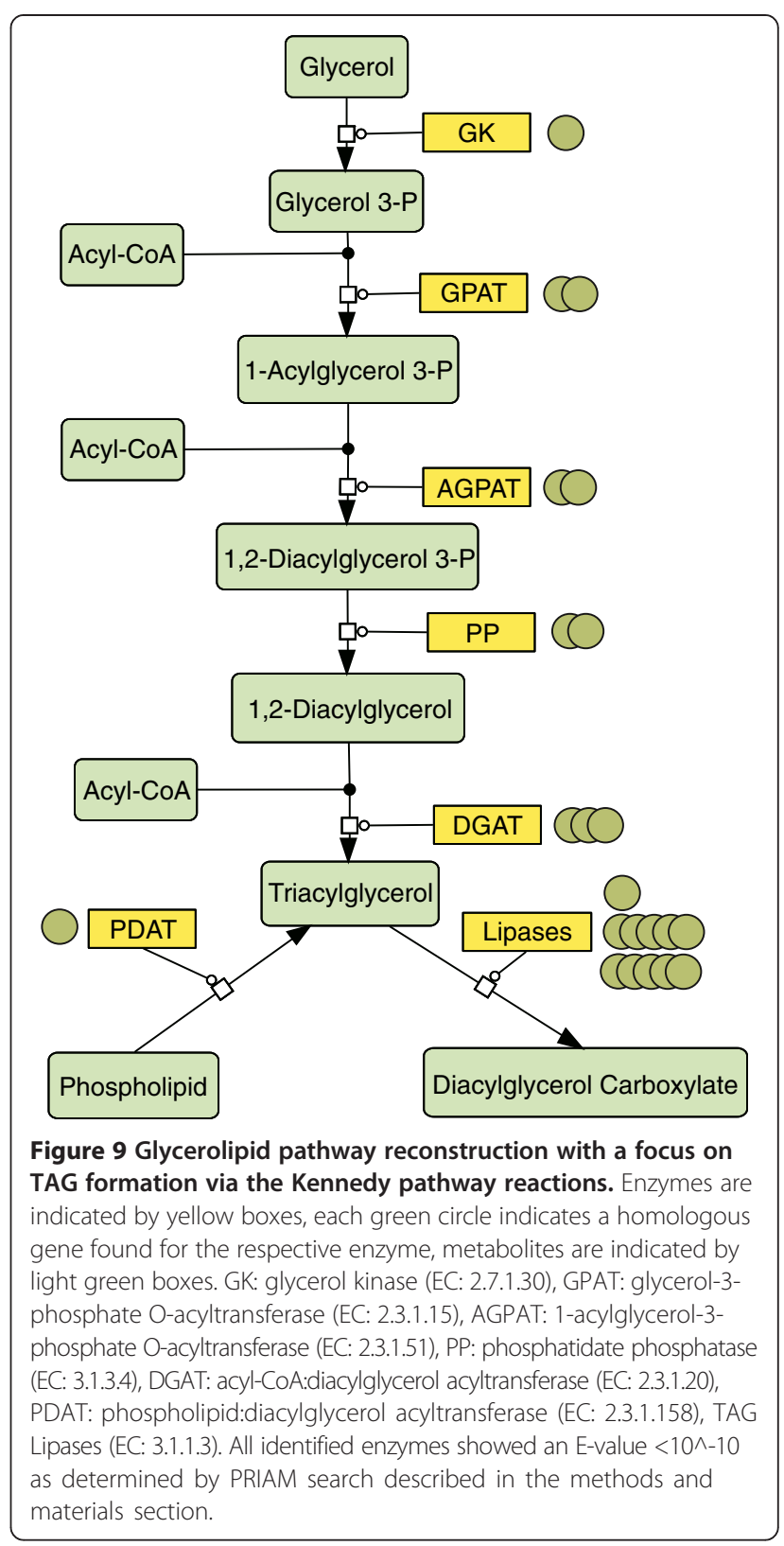

demonstrate the integrity and quality of the genome sequencing and annotation data. These results provide new insights into the lipid metabolism of green microalgae, presenting the opportunity to identify targets for future metabolic engineering that could increase the neutral lipid production potentials of M. neglectum.

\section{Discussion}

The characterization of $M$. neglectum as a robust phototrophic oleaginous strain highlights its potential suitability for oil-based biofuel production

Stress induction by nitrogen starvation and enhanced light penetration are well-known mechanisms and driving forces for increasing neutral lipid accumulation in 
many microalgal strains [2,49]. M. neglectum exhibits a distinct accumulation of neutral lipids and the fatty acids of this fraction as response to nitrogen starvation (Table 1, Figure 1B, and C). The observation that low culture densities even increase this effect (Figure 1B), underlines the importance of light intensity for reaching optimal lipid accumulations in particular for this strain.

The importance of light for optimal lipid accumulation was already demonstrated for other algae such as Nannochloropsis sp. [15] and Parachlorella kessleri [28]. However, other investigations with Chlorella minutissima and Dunaliella tertiolecta revealed no significant increase of lipids in various culture dilutions [50], suggesting species-specific differences in their glycerolipid metabolism.

Of particular note is that $M$. neglectum exhibited a comparatively high accumulation of biomass even within the nitrogen starvation period, performing clearly better than the model organism $C$. reinhardtii and the previously investigated M. contortum [19]. The neutral lipid productivity of $M$. neglectum determined in this work (52 $\mathrm{mg} \mathrm{l}^{-1}$ day $^{-1}$ ) is relatively high when compared to multiple other strains under photoautotrophic growth conditions [27], but appears low in comparison to other data considering Nannochloropsis sp. [15] and Nannochloropsis gaditana [21]. Lipid productivities of up to $204 \mathrm{mg} \mathrm{l}^{-1}$ day $^{-1}$ were reported under nitrogen deprivation for Nannochloropsis sp. [15], while N. gaditana showed productivities of up to $310 \mathrm{mg} \mathrm{l}^{-1}$ day $^{-1}$ [21]. It is however important to note that a comparison of these numbers can be misleading, since the cultivation conditions applied in the Nannochloropsis work mentioned before were considerably different to the conditions applied here, with respect to varying initial biomass densities, differences in illumination and potential stress caused by outdoor cultivation conditions. This issue is further emphasized by the fact that another Monoraphidium strain, which was suggested as potential feedstock for biodiesel production before, showed lipid productivities of $149 \mathrm{mg} \mathrm{l}^{-1} \mathrm{~d}^{-1}$ in heterotrophic and only $7 \mathrm{mg} \mathrm{l}^{-1} \mathrm{~d}^{-1}$ in autotrophic conditions [24]. For accurate evaluation of $M$. neglectum, three control strains were used in this work, C. reinhardtii, P. kessleri, and S. obliquus. Higher TAG levels were reported for S. obliquus and P. kessleri strains in recent publications in comparison to our findings $[17,51]$, indicating a clear potential to further optimize the lipid productivity of $M$. neglectum by systematic improvements of the culturing conditions, which was not in the focus of this work. As lipid productivities are highly dependent on biomass concentrations, higher productivities for $M$. neglectum are likely to be attainable through further optimization studies emphasizing optimised biomass densities and light penetration $[17,28,51]$.
The lipid contents determined for the oleaginous control strains $P$. kessleri and S. obliquus in this work were in the same range as for $M$. neglectum, demonstrating that the potential of all three species for liquid biofuels production can be regarded as being similar. C18:1 and C16:0 were the major fatty acids that accumulated in $M$. neglectum after nutrient starvation, corresponding well to previous results from the related species $M$. contortum [19], suggesting similar mechanisms of lipid accumulation in both organisms. In particular, oleic acid is known to accumulate under nitrogen starvation in many species such as Ankistrodesmus braunii, C. vulgaris, Chlorella zofingiensis, and S. obliquus [17,52]. This is in contrast to $C$. reinhardtii, where more unsaturated fatty acids are common, contributing significantly to the more diverse fatty acid pattern under nitrogen starvation. This could indicate that, in $C$. reinhardtii, membrane recycling and conversion of polar lipids to triacylglycerols plays a more prominent role [45] when compared to M. neglectum.

The high levels of C18:1 and saturated C16 in $M$. neglectum under nitrogen starvation are a promising fatty acid profile for biofuel production which encourages the cultivation of this strain in large-scale concepts for the production of biofuels, preferably in outdoor bioreactors. This composition, however, would most likely require further blending with oil from other sources to meet the requirements for liquid biofuels such as biodiesel [1].

The productivity in outdoor cultivation conditions very much relies on the robustness of the strain used. Parameters defining robustness include tolerance to changing light intensity, temperature, $\mathrm{pH}$, or salinity. Several studies have been performed on salt and $\mathrm{pH}$ tolerance of various microalgal strains [53-55]. Some adapted marine organisms such as Dunaliella tertiolecta tolerate salt concentrations up to three times higher than sea water and show an increased lipid content under these conditions [53]. These values were outstanding and could not be reached with other organisms, though some strains such as Navicula $s p$. are known to require at least salt concentrations similar to sea water to retain their highest biomass productivity [56]. In this context, M. neglectum exhibited robust phototrophic growth in salt concentrations up to $0.5 \%$ sodium chloride in the medium (Figure 2 ) which is comparatively high when compared to other freshwater strains [54]. This tolerance to salinity points towards efficient adaptation mechanisms to different salt concentrations in the investigated range. Together with the observed tolerance against high $\mathrm{pH}$ ranges (Figure 3), it is feasible to suggest that $M$. neglectum has robust growth characteristics likely suitable for outdoor cultivation.

A further distinct feature of $M$. neglectum is the increase in cell size when cultivated under brackish water 
conditions (Figure 2C) which could potentially reduce energy costs of harvesting during downstream processing [57].

Although currently no transformation strategy exists for this strain, the sequencing of its genome will allow the elucidation of regulatory regions which may become important in the construction of transformation vectors. The identification of the commonly used antibiotic hygromycin B as a selective agent within this work will also aid in future genetic manipulation attempts with this organism.

\section{The genome of $M$. neglectum reveals a highly diverse repertoire of genes encoding catalytic activities in carbohydrate and fatty acid metabolism}

For accessing the full potential of this alga as a potential biotechnological production host, the genome of $M$. neglectum was sequenced, assembled and annotated and eventually compared to existing data derived from other green microalgae and oleaginous Heterokontophyta. It could be concluded that $M$. neglectum represents a highly interesting genome which can be used for comparative analyses to further elucidate parameters for efficient neutral lipid synthesis in microalgae. With an estimated genome size of $68 \mathrm{Mbp}$ (Table 2), the M. neglectum genome is considerably larger than the genome of the Heterokontophyte Nannochloropsis gaditana (ca. $30 \mathrm{Mb},[21]$ ), but smaller than the $121 \mathrm{Mb}$ genome of the green alga C. reinhardtii [8]. However, C. reinhardtii (version 1.1) and $M$. neglectum have similar gene numbers, indicating that only a small fraction of the genome can be assigned to coding sequences. This percentage is $16.7 \%$ in C. reinhardtii [8] and slightly higher in the $M$. neglectum genome (25.1\%).

The genomic $\mathrm{G}+\mathrm{C}$ content of $64.74 \%$ of $M$. neglectum is similar to that of $C$. reinhardtii and Chlorella variabilis $[8,58]$, but considerably higher than that of $N$. gaditana (54\% [21]). Lower G+C-contents have also been reported for Cyanidioschyzon and Ostreococcus tauri (see [8], and references therein).

The size of the chloroplast was determined to be $135,362 \mathrm{bp}$, therefore, within the typical range of chloroplast genome sizes of algae and plants [59]. The gene content of the chloroplast genome is similar to that of other known algal chloroplasts. Surprisingly, the mitochondrial genome differs significantly in length from other known algal genomes. With an estimated length about $96 \mathrm{kbp}$, at present the M. neglectum mitochondrial genome represents the largest known mitochondrial genome in algae. The largest stramenopile mitochondrial genome had been identified in the diatom Phaeodactylum tricornutum (77.4 kbp, [60]). Certain plant species were shown to harbour longer mitochondrial genomes, which can reach sizes of $>2 \mathrm{Mbp}$, such as found in
Cucumis melo and Cucumis sativus [37], considerably larger than $M$. neglectum. Moreover, the mitochondrial genome of $M$. neglectum also exhibits a rather small gene density, therefore, resulting in a similar or even lower gene number than observed in other algae, including C. reinhardtii [8] or N. gaditana [21].

Research using haploid-dominant algae has for decades benefitted from ease of transformation and rapid forward and reverse genetics with $C$. reinhardtii or Volvox carteri as prominent examples [61]. In contrast, the sequenced genome of $M$. neglectum was found to be diploid, similar to several other green algae like Dunaliella salina or Haematococcus pluvialis and several diatoms [61]. This finding indicates that, compared to C. reinhardtii, future genetic transformation strategies will need to address the emergence of hemizygous individuals. Alternatively, further elucidation of the life cycle of $M$. neglectum is recommended to allow potentially easier transformation if generation of haploid cells is possible.

The total number of predicted genes in the $M$. neglectum genome is 16,845 , which is considerably higher than in $N$. gaditana (9,052 genes, [21]). A similar number of genes were predicted for C. reinhardtii (15,143 gene predictions before RNA sequencing [8]). It has to be noted that the determination of gene numbers is dependent on the prediction algorithm, its training sets, and the inclusion of other data sources such as transcriptome data. Transcriptome studies have not been performed on M. neglectum yet and are desirable to validate the predicted genes.

The differential analysis of the gene content of $M$. neglectum, C. reinhardtii, and $N$. gaditana via gene ontology (GO) allowed further insights into the degree of conservation and diversity of algal genomes. In all three investigated genomes, an over-representation of specific functional categories was found, including ATP binding, protein binding, and catalytic activity (Figure 7). These over-representations may indicate the trend to expand genomes towards regulatory mechanisms, which could be in particular the case for C. reinhardtii. Interestingly, $M$. neglectum exhibited a higher number of genes in categories related to fatty acid biosynthesis, lipid metabolic processes and carbohydrate metabolism, suggesting a higher versatility or functional redundancy when compared to the other two organisms. The reconstruction of the metabolic pathways, therefore, provided more detailed information.

The reconstruction of lipid pathways in $M$. neglectum reveals new insights into fatty acid and neutral lipid synthesis The reconstruction of the metabolic network concerning fatty acid and glycerolipid metabolism was a crucial precondition to interpret the biochemical data. This 
reconstruction was also valuable to gain important insights into the lipid metabolism of $M$. neglectum to identify potential genetic engineering targets in neutral lipid synthesis. The high number of homologous genes identified for acetyl CoA carboxylase (ACC), 3-oxoacyl-ACP reductase (KAR), and beta-ketoacyl-acyl-carrier-protein synthase (KAS1/2), suggests a more pronounced role of these factors for the lipid metabolism in $M$. neglectum compared to the green alga $C$. reinhardtii. This functional redundancy could promote the comparatively fast accumulation of fatty acids in $M$. neglectum, leading potentially to an intense use of the chloroplast pathway for producing TAG precursors.

Though the characterization of the $M$. neglectum genome also reveals the presence of a broad variety of desaturases, the fatty acid analysis of nitrogen starved cultures resulted in the identification of mainly palmitic acid (C16:0) and oleic acid (C18:1), contrasting to a high diversity of fatty acids in $C$. reinhardtii. The very distinct fatty acid production in $M$. neglectum, however, represents a very promising profile for biodiesel production from fatty acid methyl esters (FAMEs), where a high degree of saturation is desired [1]. Further analysis of the lipid metabolism identified the existence of a distinct gene coding for a palmitoyl-protein thioesterase and three further homologues for oleoyl-ACP hydrolases (Figure 8). Since thioesterases were reported to potentially reduce feedback inhibition in fatty acid biosynthesis in diatoms [62], the abundance of these enzymes could be considered as a potential bottleneck for efficient fatty acid production and consequently could be putative targets for improving the fatty acid production in microalgae by genetic engineering when genetic tools for $M$. neglectum become available.

To elucidate the role of the thioesterases in bypassing feedback inhibition, it will be of special interest to investigate their gene expression during nitrogen starvation. It has to be noted that the central carbon metabolism of diatoms shows some pronounced differences compared to green algae [63], therefore, the data from this work open up the opportunity for further comparative studies between Chlorophyta and diatoms to investigate the metabolic fluxes and their regulation towards fatty acid production, culminating in neutral lipid synthesis.

In addition to fatty acid synthesis, the abundance of triacylglcerols (TAGs) is a deciding factor for the suitability of a microalga for oil-based biofuel production.

The analysis of the $M$. neglectum genome and the reconstruction of the glycerolipid metabolism revealed the presence of only three DGAT type 1 and 2 homologs, which appears unusually low when compared to C. reinhardtii $[44,45]$ or the oleaginous Heterokontophyte N. gaditana [21]. The comparably low number of DGAT homologs indicates that this pathway for TAG generation could in vivo be less adaptive in M. neglectum.
Therefore, the DGAT genes represent highly interesting targets for comparative functional studies. Together with the distinct accumulation of $\mathrm{C} 16: 0$ and $\mathrm{C} 18: 1$ fatty acids in $M$. neglectum under nitrogen starvation, this finding furthermore points towards the important role of the chloroplast pathway for the synthesis of neutral lipid precursors [64].

\section{$M$. neglectum can serve as a model organism to elucidate the distinct role of PDAT enzymes in TAG accumulation and homeostasis}

When the acyl-Co independent mechanism of TAG generation is addressed, the presence of two PDAT homologues was confirmed with one of the candidates (Mneg_03131.t1) carrying a distinct functional acyltransferase domain. Interestingly, this is, to our knowledge, the first time that such a PDAT has been identified in the genome of an oleaginous green microalga. While PDAT homologues could be found in the transcriptome of the marine strain Dunaliella tertiolecta, they appear to be absent in Neochloris oleoabundans [43].

It is of particular note that the lipid accumulation pattern of $M$. neglectum exhibits several similarities to the oleaginous green microalga $N$. oleoabundans such as the increase of FAMEs and specifically of C18:1 (up to about 55\%), while retaining a similar percentage of C16:0 (approx. 23\%) of the fatty acid composition under nitrogen starvation [43]. Since PDAT is potentially not present or expressed in $N$. oleoabundans, a comparison of these two closely related species represents a highly interesting target for further investigations to elucidate its metabolic function at normal growth conditions and under different stresses. These findings also shed light on the diversity of lipid metabolism in the branch of green algae, as it has been noted before [11].

\section{Conclusions}

This investigation provides a foundational analysis of M. neglectum, a member of the Selenastraceae, which exhibits robust growth characteristics as well as relatively high lipid yields. Biomass and lipid production analyses presented in this work clearly demonstrate that $M$. neglectum has a high potential to serve as a production strain for future liquid biofuel concepts. This strain demonstrates favourable characteristics for cultivation including robust photoautotrophic growth, high neutral lipid contents containing an interesting profile of fatty acids during nitrogen starvation, $\mathrm{pH}$ tolerance, as well as a large cell phenotype at higher salt concentrations. A further increase of lipid yields can be expected when growth conditions are systematically optimised.

Genomic annotation and feature analysis elucidated key features of the lipid synthesis pathway, providing new insights into the evolution and physiological differences 
regarding the conversion of carbon dioxide into energydense glycerolipids in microalgae. The nuclear genome exhibits comparatively high redundancy in fatty acid synthesis processes, while the number of homologues involved in the acyl-CoA dependent TAG generation is considerably smaller, therefore, presenting potential targets for future genetic engineering approaches. The genomic analyses presented in this work will aid in the identification of potential bottlenecks for neutral lipid synthesis and provide the basis for future genetic engineering strategies.

\section{Methods}

\section{Cultivation conditions}

Monoraphidium neglectum (SAG 48.87) and Scenedesmus obliquus (SAG 276-6) were obtained from the Algae Collection in Göttingen, Germany, while Chlamydomonas reinhardtii (CC1690) was obtained from the Chlamydomonas Resource Center, University of Minnesota, US, and Parachlorella kessleri $(211 / 11 \mathrm{H})$ from the CCAP, United Kingdom. Cultivations were performed in Provasoli based minimal media (ProF) as described previously [19]. Cells were cultivated in $20 \mathrm{ml}$ minimal media in Erlenmeyer flasks on a rotary shaker and used for inoculation of 31 roller flasks adjusted to an $\mathrm{OD}_{750}$ of 0.05 . Growth was monitored by daily $\mathrm{OD}_{750}$ measurements as well as biomass development and manually counted cell number. Cultivations were performed under constant illumination with white light at intensities ranging between 350$400 \mu \mathrm{mol}$ photons $\mathrm{m}^{-2} \mathrm{~s}^{-1}$. Media were aerated with air enriched with $3 \% \mathrm{CO}_{2}$. After 3 days, cells were harvested and transferred to $400 \mathrm{ml}$ batch cultures.

\section{Nitrogen starvation and stress tolerance}

For the induction of lipid accumulation, cultures were transferred to media with the same composition but lacking a nitrogen source as described previously [19]. To assess general cultivation conditions of the second stage a further batch of 1:4 diluted cultures was transferred to nutrient replete conditions as control. Biomass was harvested after five days of cultivation, lyophilized and stored at $-80^{\circ} \mathrm{C}$ prior to further investigations.

Salt tolerance was investigated in liquid cultures containing $0.1,0.5,1.0$ and $2.0 \%(\mathrm{w} / \mathrm{v})$ sodium chloride. Performance of cultures was monitored by measurements of $\mathrm{OD}_{750}$ and the dry biomass weight. Before cultures reached the stationary phase, the biomass was harvested, lyophilized and subjected to lipid extraction and chromatographic analysis.

The $\mathrm{pH}$ sensitivity of $M$. neglectum was tested in plate assays. $5 \mathrm{ml}$ of cell suspension (adjusted to $\mathrm{OD}_{750}=0.2$ ) were spotted on agar plates containing Provasoli freshwater medium with additional $0.59 \mu \mathrm{M}$ thiamine, $4.1 \mathrm{nM}$ biotin and $0.6 \mathrm{nM}$ cobalamin. For $\mathrm{pH}$ 3.0, the Provasoli medium (adjusted to $\mathrm{pH} 3.0$ ) and agar were separately autoclaved and combined after cooling down to about $60^{\circ} \mathrm{C}$, while for pH 5.0 - 7.5 Provasoli media were adjusted to the respective $\mathrm{pH}$ before agar was added and autoclaved. For plates with $\mathrm{pH}$ 10, each stock solution for Provasoli (with $\mathrm{CaCl}_{2}$ diluted to $1 / 10$ of the original concentration) as well as double distilled water containing the agar and sodium chloride were autoclaved separately and combined at a temperature of about $60-70^{\circ} \mathrm{C}$. For mixotrophic cultivation on agar, $10 \mathrm{~g} \mathrm{~L}^{-1}$ glucose was added to media after autoclaving by filter sterilisation. Vitamins were added after media were cooled down to about $60^{\circ} \mathrm{C}$.

\section{Lipid extractions and chromatographies}

Extractions were performed in two technical replicates per biological replicate from $50 \mathrm{mg}$ lyophilized biomass. After homogenization $(3 \times 30 \mathrm{~s}$ at $6,500 \mathrm{rpm}$ using a Precellys 24, Peqlab, Erlangen, Germany), the total lipids were extracted according to a modified Folch protocol [65] using a total of $4 \mathrm{ml}$ methanol and $8 \mathrm{ml}$ chloroform. Contaminants were removed by washing the extract with $3 \mathrm{ml}$ of deionised water. From the dried total lipid extract, column chromatographies were performed to separate the neutral from the polar lipid fraction as described elsewhere [66].

\section{FAME analysis}

Neutral and polar lipids were transesterified to fatty acid methyl esters (FAME) as described previously [19]. Spectra analysis was performed with Xcalibur. The peak identity was confirmed by comparison of retention times and mass spectra to a 37 FAME mix (47885-U, Supelco). Unidentified peaks were analysed on their mass spectra and attributed to fatty acids according to their fragmentation pattern. To determine the relative amount of fatty acids within the total lipid fraction, $50 \mu \mathrm{g} \mathrm{C17-}$ triacylglycerol (glyceryl triheptadecanoate, Sigma-Aldrich, Steinheim, Germany) were added to each sample as an internal standard. Total ion chromatograms were recorded and used to calculate the relative abundances of the individual fatty acid after normalization to the internal standard (set to 100, dry biomass basis).

\section{DNA isolation and sequencing}

DNA was extracted using the cetyltrimethylammonium bromide method as reported previously [67]. After an RNAse digest (Ribonuclease A, Roth, Germany), the quality was controlled in a $1 \%$ agarose gel.

The sequencing was performed on an Illumina MiSeq machine with sequencing libraries prepared using the Illumina Nextera ${ }^{\circ}$ DNA Sample kit. DNA fragments of a size between 500 and 700 base pairs were cut from an agarose gel and purified with a MinElute Gel Extraction Kit (Qiagen). DNA amount and quality were monitored on an Agilent Bioanalyzer. The sequencing was performed 
using the MiSeq Reagent Kit v2 (Illumina) with $2 \times 250$ cycles.

\section{Genome assembly and gene annotation}

All reads obtained by genome sequencing were assembled to contigs and scaffolds using the Newbler assembler version 2.6 (Roche) with settings for heterozygous genomes. Nuclear and organelle genomes were assembled manually using the compatible finishing package Consed version 23.0 [68].

The annotation of the three genomes (chloroplast, mitochondrion and nuclear) was done by a specific annotation pipeline, which consists of three steps. All potential genes were predicted by two $a b$ initio gene prediction tools: Augustus [31] with the Chlamydomonas reinhardtii genome as training set and in parallel with GeneMark-ES (version 2) [32], which combines GeneMark.hmm for prediction of eukaryotic genomes with a self-training procedure. In addition, a protein alignment with all $C$. reinhardtii proteins was performed. To evaluate over 34,000 predicted genes the software EVidenceModeler (EVM) [33] was used to filter the gene set and to eliminate putative false positive predictions. For that purpose we assigned different weightings for the different prediction outputs. Augustus and GeneMark were assigned with the same higher weight (2) and the protein alignment a lower weight (1). Ribosomal RNAs were identified by using the RNAmmer 1.2 server [69] and tRNAs were determined by tRNAscan-SE version 1.21 [70,71]. Organellar genomes were analysed and printed with OGDRAW [30]. All gene information were integrated to the annotation platform GenDBE, which is a modification of GenDB for the annotation of eukaryotic genomes [34]. GenDBE allows the manual curation of gene specific annotation in addition to the visualisation of gene order.

This Whole Genome Shotgun project has been deposited at DDBJ/EMBL/GenBank under the accession AYTC00000000. The version described in this paper is version AYTC01000000.

For genome comparison based on $\mathrm{GO}$ categories gene specific GO-terms were obtained by analysing all genes of the three genomes $C$. reinhardtii, $N$. gaditana and $M$. neglectum in Interpro [41].

\section{Phylogenetic analysis}

The evolutionary history was inferred by using the Maximum Likelihood method based on the Tamura-Nei model [72], with the tree of the highest log likelihood (-6385.4692) shown. Initial trees were obtained by the Neighbor-Joining method to a matrix of pairwise distances, estimated using the Maximum Composite Likelihood (MCL) approach. The tree is drawn to scale. The branch lengths are measured in number of substitutions per site. Numbers indicate bootstrap values after 5,000 replications. The analysis involved 12 nucleotide sequences with a total of 1284 positions in the final dataset. Evolutionary analyses were conducted in MEGA5 [73].

\section{Metabolic pathway reconstruction}

Pathway reconstruction was performed for FA and TAG synthesis by means of PathwayTools and PRIAM. The KEGG pathway proposed on the gene set of $M$. neglectum by GenDBE was analysed using PRIAM (database profile of March 2013) [34,74]. Therefore, the E.C. numbers of the proposed KEGG pathway were used as basis for the PRIAM search to identify corresponding genes with an E-value cutoff of $1 * 10^{-10}$.

\section{Additional files}

Additional file 1: Figure S1. Neutral lipid productivity of $M$. neglectum when compared to $C$. reinhardtii for the overall production period: 3 days growth under nutrient replete conditions $\left(\mathrm{OD}_{750}=0.05-0.06\right.$ for inoculation), 5 days starvation under nitrogen deficiency ( $+\mathrm{N}$, preculture and $-\mathrm{N}$, high OD).

Additional file 2: Figure S2. Fatty acid abundances of $M$. neglectum and $C$. reinhardtii grown under nutrient replete $(+N)$ and nitrogen starvation (-N) conditions as determined via GC-MS. Error bars show standard deviation $(n=4)$.

Additional file 3: Figure S3. Read versus contig plot reveals diploid character of the $M$. neglectum genome.

Additional file 4: Figure S4. Comparison of chloroplast genomes of C. reinhardtii, M. neglectum and N. gaditana.

\section{Abbreviations}

ACP: Acyl carrier protein; BLAST: Basic local alignment search tool; CoA: Coenzyme A; DAG: Diacylglycerol; DNA: Deoxyribonucleic acid; EVM: EVidence modeler; FA: Fatty acid; $\mathrm{NaCl}$ : Sodium chloride; TAG: Triacylglycerol; TRIS: Tris(hydroxymethyl)aminomethane.

\section{Competing interests}

The authors declare that they have no competing interests.

\section{Authors' contributions}

The research was designed by $C B, A A D, J H M$, JK and OK. CB, AAD, JW, MG, $\mathrm{KL}$, OBK performed the research, with the main part of this work carried out by the first author, CB. CB performed growth analysis, lipid analysis and FAME profiling under nutrient replete conditions and nitrogen starvation, was involved in genome sequencing, performed phylogenetic analysis, and in silico reconstruction of lipid metabolism, prepared therefore figures and tables and drafted the manuscript. JW investigated growth under nitrogen starvation and performed lipid analysis. MG performed investigations of salt tolerance, KJL of resistance to antibiotics and OBK of pH tolerance. Genome sequencing was performed by $\mathrm{AA}$, genome assembly and annotation by AA and AAD. The GENDBE project was created and maintained by OR and AG. Manual gene assignments and comparison of genomes was carried out by AAD. AAD prepared figures and tables related to genome assembly and metabolic reconstruction. Data analysis and interpretation: $C B, A A D, J H M, O K$. Manuscript writing: $C B, A A D, J K, J H M, O K$. All authors read and approved the final manuscript.

\section{Acknowledgements}

This work was supported by Neste Oil Oyj and the Ministry of Science, Innovation \& Research of the German State of North Rhine-Westphalia via the CLIB Graduate Cluster Industrial Biotechnology.

Received: 25 July 2013 Accepted: 19 December 2013

Published: 28 December 2013 
References

1. Schenk PMP, Thomas-Hall SR, Evan S, Marx U, Mussgnug JH, Posten C, Kruse O, Hankamer B: Second generation biofuels: high-efficiency microalgae for biodiesel production. BioEnergy Res 2008, 1(1):20-43.

2. Hu Q, Sommerfeld M, Jarvis E, Ghirardi M, Posewitz M, Seibert M, Darzins A Microalgal triacylglycerols as feedstocks for biofuel production: perspectives and advances. Plant J 2008, 54(4):621-639.

3. Chisti Y: Biodiesel from microalgae. Biotechnol Adv 2007, 25(3):294-306.

4. Spolaore $P$, Joannis-Cassan C, Duran E, Isambert A: Commercial applications of microalgae. Soc Biotechnol Japan 2006, 101(2):87-96.

5. Mata TM, Martins ANA, Caetano NS: Microalgae for biodiesel production and other applications: A review. Renew Sust Energ Rev 2010, 14(1):217-232

6. Vieler A, Wu G, Tsai C-H, Bullard B, Cornish AJ, Harvey C, Reca I-B, Thornburg C Achawanantakun R, Buehl CJ, et al: Genome, functional gene annotation, and nuclear transformation of the Heterokont oleaginous alga Nannochloropsis oceanica CCMP1779. PLoS Genet 2012, 8(11):e1003064.

7. Armbrust EV, Berges JA, Bowler C, Green BR, Martinez D, Putnam NH, Zhou S, Allen AE, Apt KE, Bechner $M$, et al: The genome of the Diatom Thalassiosira pseudonana: ecology, evolution, and metabolism. Science 2004, 306(5693):79-86.

8. Merchant SS, Prochnik SE, Vallon O, Harris EH, Karpowicz SJ, Witman GB, Terry A, Salamov A, Fritz-Laylin LK, Marechal-Drouard L, et al: The Chlamydomonas genome reveals the evolution of key animal and plant functions. Science 2007, 318(5848):245-250.

9. Gong $Y$, Jiang M: Biodiesel production with microalgae as feedstock: from strains to biodiesel. Biotechnol Lett 2011, 33(7):1269-1284.

10. Li Y, Han D, Hu G, Sommerfeld M, Hu Q: Inhibition of starch synthesis results in overproduction of lipids in Chlamydomonas reinhardtii. Biotechnol Bioeng 2010, 107(2):258-268.

11. Liu B, Benning C: Lipid metabolism in microalgae distinguishes itself. Curr Opin Biotechnol 2013, 24(2):300-309.

12. Harwood JL, Guschina IA: The versatility of algae and their lipid metabolism. Biochimie 2009, 91(6):679-684

13. Lang I, Hodac L, Friedl T, Feussner I: Fatty acid profiles and their distribution patterns in microalgae: a comprehensive analysis of more than 2000 strains from the SAG culture collection. BMC Plant Biol 2011, 11(1):124.

14. Lim DKY, Garg S, Timmins M, Zhang ESB, Thomas-Hall SR, Schuhmann H, Li Y, Schenk PM: Isolation and evaluation of oil-producing microalgae from subtropical coastal and brackish waters. PLOS ONE 2012, 7(7):e40751.

15. Rodolfi L, Zittelli GC, Bassi N, Padovani G, Biondi N, Bonini G, Tredici MR: Microalgae for oil: strain selection, induction of lipid synthesis and outdoor mass cultivation in a low-cost photobioreactor. Biotechnol Bioeng 2009, 102(1):100-112.

16. MacDougall K, McNichol J, McGinn P, O'Leary SB, Melanson J: Triacylglycerol profiling of microalgae strains for biofuel feedstock by liquid chromatography-high-resolution mass spectrometry. Anal Bioanal Chem 2011, 401(8):2609-2616.

17. Breuer G, Lamers PP, Martens DE, Draaisma RB, Wijffels RH: The impact of nitrogen starvation on the dynamics of triacylglycerol accumulation in nine microalgae strains. Bioresour Technol 2012, 124:217-226.

18. Griffiths M, van Hille R, Harrison S: Lipid productivity, settling potential and fatty acid profile of 11 microalgal species grown under nitrogen replete and limited conditions. J Appl Psychol 2012, 245(5):989-1001.

19. Bogen C, Klassen V, Wichmann J, Russa ML, Doebbe A, Grundmann M Uronen P, Kruse O, Mussgnug JH: Identification of Monoraphidium contortum as a promising species for liquid biofuel production. Bioresour Technol 2013, 133:622-626.

20. Jinkerson RE, Radakovits R, Posewitz MC: Genomic insights from the oleaginous model alga Nannochloropsis gaditana. Bioengineered 2013 4(1):37-43.

21. Radakovits $R$, Jinkerson RE, Fuerstenberg SI, Tae H, Settlage RE, Boore JL, Posewitz MC: Draft genome sequence and genetic transformation of the oleaginous alga Nannochloropis gaditana. Nat Commun 2012, 3:686.

22. Sasso S, Pohnert G, Lohr M, Mittag M, Hertweck C: Microalgae in the postgenomic era: a blooming reservoir for new natural products. FEMS Microbiol Rev 2012, 36(4):761-785.

23. Chaichalerm S, Pokethitiyook P, Yuan W, Meetam M, Sritong K, Pugkaew W, Kungvansaichol K, Kruatrachue M, Damrongphol P: Culture of microalgal strains isolated from natural habitats in Thailand in various enriched media. App/ Energy 2012, 89(1):296-302.
24. Yu X, Zhao P, He C, Li J, Tang X, Zhou J, Huang Z: Isolation of a novel strain of Monoraphidium sp. and characterization of its potential application as biodiesel feedstock. Bioresource Technol 2012, 121:256-262

25. Krienitz L, Ustinova I, Friedl T, Huss VAR: Traditional generic concepts versus $18 \mathrm{~S}$ rRNA gene phylogeny in the green algal family Selenastraceae (Chlorophyceae, Chlorophyta). J Phycol 2001, 37(5):852-865.

26. Griffiths MJ, Harrison STL: Lipid productivity as a key characteristic for choosing algal species for biodiesel production. J Appl Phycol 2009, 21(5):493-507

27. Chen C-Y, Yeh K-L, Aisyah R, Lee D-J, Chang J-S: Cultivation, photobioreactor design and harvesting of microalgae for biodiesel production: A critical review. Bioresource Technol 2011, 102(1):71-81.

28. Li X, Přibyl P, Bišová K, Kawano S, Cepák V, Zachleder V, Čižková M Brányiková I, Vítová M: The microalga Parachlorella kessleri--A novel highly efficient lipid producer. Biotechnol Bioeng 2012, 110(1):97-107.

29. Wibberg D, Jelonek L, Rupp O, Hennig M, Eikmeyer F, Goesmann A, Hartmann A, Borriss R, Grosch R, Pühler A, et al: Establishment and interpretation of the genome sequence of the phytopathogenic fungus Rhizoctonia solani AG1-IB isolate 7/3/14. J Biotechnol 2013, 167(2):142-155.

30. Lohse M, Drechsel O, Bock R: OrganellarGenomeDRAW (OGDRAW): a tool for the easy generation of high-quality custom graphical maps of plastid and mitochondrial genomes. Curr Genetics 2007, 52(5-6):267-274

31. Stanke M, Waack S: Gene prediction with a hidden Markov model and a new intron submodel. Bioinformatics 2003, 19(suppl 2):ii215-ii225.

32. Borodovsky M, Lomsadze A: Eukaryotic gene prediction using GeneMark. hmm-E and GeneMark-ES. In Current Protocols in Bioinformatics. John Wiley \& Sons, Inc; 2002

33. Haas BJ, Salzberg SL, Zhu W, Pertea M, Allen JE, Orvis J, White O, Buell CR, Wortman JR: Automated eukaryotic gene structure annotation using EVidenceModeler and the Program to Assemble Spliced Alignments. Genome Biol 2008, 9(1):R7.

34. Meyer F, Goesmann A, McHardy AC, Bartels D, Bekel T, Clausen J, Kalinowski J, Linke B, Rupp O, Giegerich R: GenDB-an open source genome annotation system for prokaryote genomes. Nucleic Acids Res 2003, 31(8):2187-2195.

35. Simpson CL, Stern DB: The treasure trove of algal chloroplast genomes. Surprises in architecture and gene content, and their functional implications. Plant Physiol 2002, 129(3):957-966.

36. Odintsova MS, Yurina NP: Genomics and evolution of cellular organelles. Russ J Genet 2005, 41(9):957-967.

37. Nash EA, Nisbet RER, Barbrook AC, Howe CJ: Dinoflagellates: a mitochondrial genome all at sea. Trends Genet 2008, 24(7):328-335.

38. Waller RF, Jackson CJ: Dinoflagellate mitochondrial genomes: stretching the rules of molecular biology. Bioessays 2009, 31(2):237-245.

39. Blom J, Albaum SP, Doppmeier D, Puhler A, Vorholter FJ, Zakrzewski M, Goesmann A: EDGAR: A software framework for the comparative analysis of prokaryotic genomes. Bmc Bioinformatics 2009, 10:154.

40. Mandal S, Mallick N: Microalga Scenedesmus obliquus as a potential source for biodiesel production. Appl Microbiol Biotechnol 2009, 84(2):281-291

41. Hunter S, Jones $P$, Mitchell A, Apweiler R, Attwood TK, Bateman A, Bernard T, Binns D, Bork P, Burge $S$, et al: InterPro in 2011: new developments in the family and domain prediction database. Nucleic Acids Res 2012, 40(D1):D306-D312.

42. Wada H, Murata N, Moellering ER, Miller R, Benning C: Molecular genetics of lipid metabolism in the model green alga Chlamydomonas reinhardtii. In Lipids in Photosynthesis, Volume 30. Edited by Govindjee. Netherlands: Springer; 2009:139-155.

43. Rismani-Yazdi H, Haznedaroglu BZ, Hsin C, Peccia J: Transcriptomic analysis of the oleaginous microalga Neochloris oleoabundans reveals metabolic insights into triacylglyceride accumulation. Biotechnol Biofuels 2012, 5:74.

44. La Russa M, Bogen C, Uhmeyer A, Doebbe A, Filippone E, Kruse O, Mussgnug JH: Functional analysis of three type-2 DGAT homologue genes for triacylglycerol production in the green microalga Chlamydomonas reinhardtii. J Biotechnol 2012, 162(1):13-20.

45. Boyle NR, Page MD, Liu B, Blaby IK, Casero D, Kropat J, Cokus S, Hong-Hermesdorf A, Shaw J, Karpowicz SJ, et al: Three acyltransferases and a nitrogen responsive regulator are implicated in nitrogen starvation-induced triacylglycerol accumulation in Chlamydomonas. J Biol Chem 2012, 287(19):15811-15825

46. Andrianov V, Borisjuk N, Pogrebnyak N, Brinker A, Dixon J, Spitsin S, Flynn J, Matyszczuk P, Andryszak K, Laurelli M, et al: Tobacco as a production 
platform for biofuel: overexpression of Arabidopsis DGAT and LEC2 genes increases accumulation and shifts the composition of lipids in green biomass. Plant Biotechnol J 2010, 8(3):277-287.

47. Hildebrand M, Abbriano RM, Polle JEW, Traller JC, Trentacoste EM, Smith SR, Davis AK: Metabolic and cellular organization in evolutionarily diverse microalgae as related to biofuels production. Curr Opin Chem Biol 2013, 17:506-514

48. Yoon K, Han D, Li Y, Sommerfeld M, Hu Q: Phospholipid:diacylglycerol acyltransferase is a multifunctional enzyme involved in membrane lipid turnover and degradation while synthesizing triacylglycerol in the unicellular green microalga Chlamydomonas reinhardtii. The Plant Cell Online 2012, 24(9):3708-3724.

49. Solovchenko A: Physiological role of neutral lipid accumulation in eukaryotic microalgae under stresses. Russian J Plant Physiol 2012, 59(2):167-176

50. Tang $\mathrm{H}$, Chen M, Simon Ng KY, Salley SO: Continuous microalgae cultivation in a photobioreactor. Biotechnol Bioengineer 2012 109(10):2468-2474.

51. Pribyl P, Cepák V, Zachleder V: Production of lipids in 10 strains of Chlorella and Parachlorella, and enhanced lipid productivity in Chlorella vulgaris. Appl Microbiol Biotechnol 2012, 94(2):549-561.

52. Williams VR, McMillan R: Lipids of Ankistrodesmus braunii. Science 1961, 133(3451):459-460.

53. Takagi M, Karseno Y, Yoshida T: Effect of salt concentration on intracellular accumulation of lipids and triacylglyceride in marine microalgae Dunaliella cells. J Biosci Bioengineer 2006, 101(3):223-226.

54. Salama E-S, Kim H-C, Abou-Shanab Rl, Ji M-K, Oh Y-K, Kim S-H, Jeon B-H: Biomass, lipid content, and fatty acid composition of freshwater Chlamydomonas mexicana and Scenedesmus obliquus grown under salt stress. Bioproc Biosyst Engineer 2013:1-7.

55. Santos AM, Janssen M, Lamers PP, Evers WAC, Wijffels RH: Growth of oil accumulating microalga Neochloris oleoabundans under alkaline-saline conditions. Bioresource Technol 2012, 104:593-599.

56. Matsumoto M, Sugiyama H, Maeda Y, Sato R, Tanaka T, Matsunaga T: Marine Diatom, Navicula sp. strain JPCC DA0580 and marine green alga, Chlorella sp. strain NKG400014 as potential sources for biodiesel production. Appl Biochem Biotechnol 2010, 161(1):483-490.

57. Milledge J, Heaven S: A review of the harvesting of micro-algae for biofuel production. Rev Environ Sci Biotechnol 2013, 12(2):165-178.

58. Blanc G, Duncan G, Agarkova I, Borodovsky M, Gurnon J, Kuo A, Lindquist E, Lucas S, Pangilinan J, Polle J, et al: The Chlorella variabilis NC64A genome reveals adaptation to photosymbiosis, coevolution with viruses, and cryptic sex. Plant Cell 2010, 22(9):2943-2955.

59. Chan CX, Gross J, Yoon HS, Bhattacharya D: Plastid origin and evolution: new models provide insights into old problems. Plant Physiol 2011, 155(4):1552-1560.

60. Oudot-Le Secq M-P, Green BR: Complex repeat structures and novel features in the mitochondrial genomes of the diatoms Phaeodactylum tricornutum and Thalassiosira pseudonana. Gene 2011, 476(1-2):20-26.

61. Coll J: Review. Methodologies for transferring DNA into eukaryotic microalgae. Span J Agric Res 2006, 4(4):316-330.

62. Gong Y, Guo X, Wan X, Liang Z, Jiang M: Characterization of a novel thioesterase (PtTE) from Phaeodactylum tricornutum. J Basic Microbiol 2011, 51(6):666-672.

63. Hockin NL, Mock T, Mulholland F, Kopriva S, Malin G: The response of Diatom central carbon metabolism to nitrogen starvation is different from that of green algae and higher plants. Plant Physiol 2012, 158(1):299-312.

64. Fan J, Andre C, Xu C: A chloroplast pathway for the de novo biosynthesis of triacylglycerol in Chlamydomonas reinhardtii. FEBS lett 2011, 585(12): 1985-1991

65. Folch J, Lees M, Stanley GHS: A simple method for the isolation and purification of total lipides from animal tissues. J Biol Chem 1957, 226(1):497-509.

66. Li Y, Han D, Hu G, Dauvillee D, Sommerfeld M, Ball S, Hu Q: Chlamydomonas starchless mutant defective in ADP-glucose pyrophosphorylase hyperaccumulates triacylglycerol. Metab Engineer 2010, 12(4):387-391.

67. Murray MG, Thompson WF: Rapid isolation of high molecular weight plant DNA. Nucleic Acids Res 1980, 8(19):4321-4326.

68. Gordon D: Viewing and Editing Assembled Sequences Using Consed. In Current Protocols in Bioinformatics. John Wiley \& Sons, Inc; 2003:2:11.2.1-11.2.43.
69. Lagesen K, Hallin P, Rødland EA, Stærfeldt H-H, Rognes T, Ussery DW: RNAmmer: consistent and rapid annotation of ribosomal RNA genes. Nucleic Acids Res 2007, 35(9):3100-3108.

70. Schattner P, Brooks AN, Lowe TM: The tRNAscan-SE, snoscan and snoGPS web servers for the detection of tRNAs and snoRNAs. Nucleic Acids Res 2005, 33(suppl 2):W686-W689.

71. Lowe TM, Eddy SR: tRNAscan-SE: a program for improved detection of transfer RNA genes in genomic sequence. Nucleic Acids Res 1997, 25(5):0955-0964

72. Tamura K, Nei M: Estimation of the number of nucleotide substitutions in the control region of mitochondrial DNA in humans and chimpanzees. Mole Biol Evol 1993, 10(3):512-526.

73. Tamura K, Peterson D, Peterson N, Stecher G, Nei M, Kumar S: MEGA5: Molecular evolutionary genetics analysis using maximum likelihood, evolutionary distance, and maximum parsimony methods. Mole Biol Evol 2011, 28(10):2731-2739.

74. Claudel-Renard C, Chevalet C, Faraut T, Kahn D: Enzyme-specific profiles for genome annotation: PRIAM. Nucleic Acids Res 2003, 31(22):6633-6639.

doi:10.1186/1471-2164-14-926

Cite this article as: Bogen et al:: Reconstruction of the lipid metabolism for the microalga Monoraphidium neglectum from its genome sequence reveals characteristics suitable for biofuel production. BMC Genomics 2013 14:926

\section{Submit your next manuscript to BioMed Central and take full advantage of:}

- Convenient online submission

- Thorough peer review

- No space constraints or color figure charges

- Immediate publication on acceptance

- Inclusion in PubMed, CAS, Scopus and Google Scholar

- Research which is freely available for redistribution 